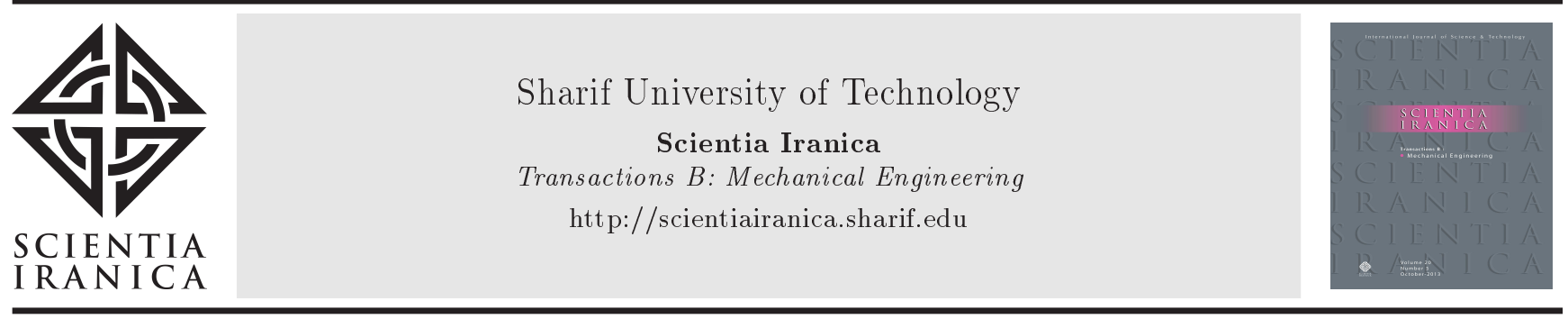

\title{
Optimized design of adaptable vibrations suppressors in semi-active control of circular plate vibrations
}

\author{
N. Asmari Saadabad, H. Moradi*, and G.R. Vossoughi \\ Centre of Excellence in Design, Robotics \& Automation, Department of Mechanical Engineering, Sharif University of Technology, \\ Tehran, Iran.
}

Received 28 May 2017; received in revised form 20 November 2017; accepted 9 April 2018

\section{KEYWORDS \\ Circular plates; \\ Adaptable vibration \\ suppressor; \\ Semi-active control; \\ Advanced algorithm; \\ Optimal design.}

\begin{abstract}
Due to flexibility of thin plates, high-amplitude vibrations are observed when they are subjected to severe dynamic loads. Because of the extensive application of circular plates in industry, attenuating the undesired vibrations is of foremost importance. In this paper, Adaptable Vibration Suppressors (AVSs) as a semi-active control approach were utilized to suppress the vibrations in a free circular plate under the concentrative harmonic excitation. Using mode summation method, the mathematical model of the hybrid system including the plate and an arbitrary number of vibration suppressors was analyzed. By developing a complex multiple-loop algorithm, optimum values for the parameters of AVSs (stiffness and position) were achieved such that the plate deflection was comprehensively minimized. According to the results, AVSs acted efficiently in suppressing the vibrations in resonance/non-resonance conditions. It was also observed that optimum AVSs reduced the plate deflection over a broad spectrum of excitation frequencies. Finally, since the algorithm was developed in a general user friendly style, design of AVSs could be extended to other shapes of plates with various boundary conditions and excitations.
\end{abstract}

(C) 2019 Sharif University of Technology. All rights reserved.

\section{Introduction}

In recent years, continuous circular plates have extensively been used in complex engineering structures. Manufacturing processes, aeronautics, marine structures, pressure vessels, rocket launching pads, and instrument mounting bases for space vehicles are some of the instances in which these plates are used [1]. In most of these structures, the plates are subjected to severe dynamic loadings, which induce large vibration amplitudes due to their flexibility.

\footnotetext{
*. Corresponding author. Tel.: +9821661655545; Fax: +98 2166000021

E-mail addresses: hamedmoradi@sharif.edu and hamedmoradi@asme.org (H. Moradi).
}

Although the dynamics of the circular plates are described through complex governing equations, the vibrations induced by conservative and non-conservative loads have widely been studied. Following the attempts to find the natural frequencies of circular plates, Minkarah and Hoppmann [2] obtained series solutions for simply supported and clamped circular plates. Narita [3] utilized the Rayleigh-Ritz method to derive the power series of the vibration equations, while Kang et al. [4] obtained approximate closed-form solutions for free vibrations of circular plates. Exact solutions for free vibrations of circular plates with various boundary conditions including free, clamped, and simply supported were presented by Leissa $[5,6]$. In the early stages, several researches were carried out to study the axisymmetric vibration of circular plates. For instance, Yamaki [7], and Kung and Pao [8] analyzed the one-mode vibration with various boundary condi- 
tions while they neglected damping effects. Moreover, in regard to the rotating circular discs, Tobias, Arnold, and Williams [9-11] took the asymmetric modes into account. Recently, Shi et al. [12] proposed a unified approach to vibration analysis of circular plates under arbitrary boundary conditions.

In parallel with the free vibrations of circular plates, their forced response behavior was also studied. A thorough research was conducted by Sridhar et al. [13] who obtained the forced response of a clamped circular plate considering the effects of damping. With the introduction of computer methods, new studies were performed. Combining the Finite Element Method (FEM) and harmonics balance approach, Ribeiro and Petyt $[14,15]$ conducted numerical research with computer simulations. Wu et al. [16] analyzed static and free vibrations of plates using hybrid smoothed FEM. Later, effects of harmonic excitations were studied in several studies. For instance, Haterbouch and Benamar [17,18] executed some investigations into the harmonic non-linear vibrations of circular plates. Recently, Liou [19] studied the effect of time-dependent excitation on circular plate, numerically.

Non-conservative loads induce two main instabilities in plates: divergence and flutter. Through monitoring dynamic system vibrations, the flutter type of instability can be detected. As it is well-known, under resonance conditions, large vibration amplitudes are observed. Consequently, the plate is vulnerable to fatigue or fractures in severe dynamic loading cases. Moreover, the necessity of using thin plates in reducing the mass to its maximum extent increases the flexibility of the plates. In order to suppress the high-amplitude vibrations, methods to control and attenuate the harmful vibrations are required.

Many passive and active control approaches have been used to suppress large vibration amplitudes of circular plates. Kerlin [20] analyzed the effect of a plate-like dynamic vibration absorber. Initially, this type of absorber was introduced by Snowdon [21]. Kirk and Leissa [22] examined how a concentric ring-stiffener affected the lowest vibration frequencies of a circular plate and tried to find the best position to place the absorber. Azimi [23] presented a way to attenuate the vibrations using an elastic point support on the plate. Kunukkasseril and Swamidas [24] investigated the free vibrations of isotropic and orthotropic plates supported by a finite number of elastic or rigid concentric rings. Avalos et al. [25] found the approximate solution for the vibrations of a circular plate carrying an elastically mounted mass by using the optimized Rayleigh-Ritz method.

Among the active methods for controlling the vibrations, some studies have appeared to work efficiently. Ray and Shivakumar [26] tested the method of using Active Constrained Layer Damping (ACLD) to suppress the vibrations in thin plates. Vidoli and Dell'Isola [27] implemented electrically interconnected piezoelectric actuators to attenuate the vibrations while Caruso et al. [28] placed couples of piezoelectric patches, used as sensors and actuators, to attenuate the oscillations. Wu et al. [29] incorporated a linear motor to control the vibrations. Qiu et al. [30] analyzed the effect of piezoelectric ceramics patches as sensors and actuators to suppress the vibration of the smart flexible clamped plate. In the study by $\mathrm{Hu}$ and $\mathrm{Ng}$ [31], active robust vibration control method was tested via experimental implementation to reduce the vibrations in circular structures. Wiciak [32] did also make use of piezoelectric actuators for active vibration suppression and analyzed the results numerically. In a recent study, Khorshidi et al. [33] applied an active control method to attenuate the transverse vibration of plates under harmonic loads. Several efforts have been put into attenuating the vibrations by utilizing smart materials. Among them, application of adaptive synchronized switch damping on voltage to composite beams [34] and semi-passive piezoelectric-based control with synchronized switch damping method [35] are of foremost importance.

Although various passive/active control methods have been implemented, there are still some deficiencies. The passive control methods may not be able to suppress vibrations over a broad spectrum of excitation frequencies. This is because with a low number of suppressors, it is not possible to suppress complex mode shapes of the plate. Although active control methods have been designed for a wide range of frequencies, few studies have investigated their use in circular plates. According to the literature, active methods are essentially applied to rectangular plates. Furthermore, the active methods usually require expensive mechanisms to work effectively on thin plates. Finally, it should be noticed that in the majority of previous researches, passive absorbers or actuators have been fixed in specific positions. In the presence of complex mode shapes of circular plates, this limitation is regarded as a hindrance to suppressing the vibrations.

Attenuating the vibrations in plates that are subject to harmonic loads can broaden the applications of circular plates in industry, dramatically. Since Adaptable Vibration Suppressors (AVSs) can strengthen resistance of the circular plates to a broad range of excitation frequencies, circular-shape structures that are comparatively easy to manufacture can incorporate large fatigue-resistant or fracture-resistant plates. Marine vehicles, circular tanks or pipes in which the fluid is in dynamic motion, and vibrant-instrument mounting bases are some of the frameworks that can be established using circular shaped plates.

In this paper, optimized Adaptable Vibration 
Suppressors (AVSs) are designed to suppress the vibrations in the circular plate that are caused by harmonic excitation of the center point. Saadabad et al. [36] showed that implementing tunable vibration absorbers could reduce the undesired oscillations in transmission line as a continuous system over a wide range of excitation frequencies. Saadabad et al. [36] investigated the stiffness and position of absorbers in a one-dimensional space. In the present work, the problem is extended to 2-dimensional space. This adds to the complexity of the proposed algorithm; while the distribution of the natural frequencies is various in a circular plate, the transmission line is modelled as a 1-dimensional string. In the presented semi-active control method, an iterative multiple-loop optimization algorithm is established and utilized to determine the optimum number of AVSs and best values of their parameters (stiffness and locus) such that the circular plate deflection is minimized. By analyzing the problem in the time/frequency domains, an efficient look-up diagram for the design of AVSs is developed for a broad range of excitation frequencies. It is shown that implementing passive vibration suppressors that are placed on the structure based on an active design process can be efficient in reducing the oscillations. Implementing the look-up diagram with adaptive-stiffness suppressors and displacing the suppressors based on the external excitation provide the means for a semi-active vibration control of the structure.

\section{Modeling the combined dynamical system of circular plate and finite number of adaptable vibration suppressors}

The model of the circular plate under a harmonic excitation force is presented in Figure 1. The plate is modeled as a continuous system with infinite number of elements. In a given domain, the general equation of motion for the plate is described as [37]:

$$
m(\aleph) \ddot{w}(\aleph, t)+L w(\aleph, t)=f(\aleph, t),
$$

in which $\aleph$ is a point in the domain, $w(\aleph, t)$ is the deflection of that point at time $t, L$ is a stiffness differential operator with linear homogenous self-adjoint characteristics, $m(\aleph)$ represents the mass density, and $f(\aleph, t)$ is the force density at point $\aleph$. If the normal modes of the circular plate, $W_{m n}(r, \theta)$, are known, its deflection at any point can be written in the form:

$$
w(r, \theta, t)=\sum_{m=0}^{\infty} \sum_{n=1}^{\infty} W_{m n}(r, \theta) \eta_{m n}(t),
$$

in which, $\eta_{m n}(t)$ is the time-dependent modal coordinate. Substituting Eq. (2) in Eq. (1), and defining the generalized forces as:

$$
N_{m n}(t)=\int_{0}^{2 \pi} \int_{0}^{R} W_{m n}(r, \theta) f(r, \theta, t) r d r d \theta,
$$

yields the modal equation in the generalized coordinates:

$$
\ddot{\eta}_{m n}(t)+\omega_{m n}^{2} \eta_{m n}(t)=N_{m n}(t),
$$

where, in Eq. (3), $f(r, \theta, t)=F(t) \delta(r-\bar{r}, \theta-\bar{\theta})$ is the distribution of force on the plate. Taking the damping effects of the plate into account (dented by $\zeta_{m n}$ ), the modal equation is modified as:

$$
\ddot{\eta}_{m n}(t)+2 \zeta_{m n} \omega_{m n} \dot{\eta}_{m n}(t)+\omega_{m n}^{2} \eta_{m n}(t)=N_{m n}(t) .
$$

The normal mode shapes in a circular domain given by $0<r<R$, are found based on the differential equation [29]:

$$
\nabla^{4} W(r, \theta)-\beta^{4} W(r, \theta)=0, \quad \beta^{4}=\frac{m \omega^{2}}{D_{E}},
$$

where $D_{E}=\frac{E h^{3}}{12\left(1-\nu^{2}\right)}$ is the plate flexural rigidity, $E$ is Young's modulus, $h$ is the plate thickness, and $\nu$ is Poisson's ratio. The solution to Eq. (6) is in the form:

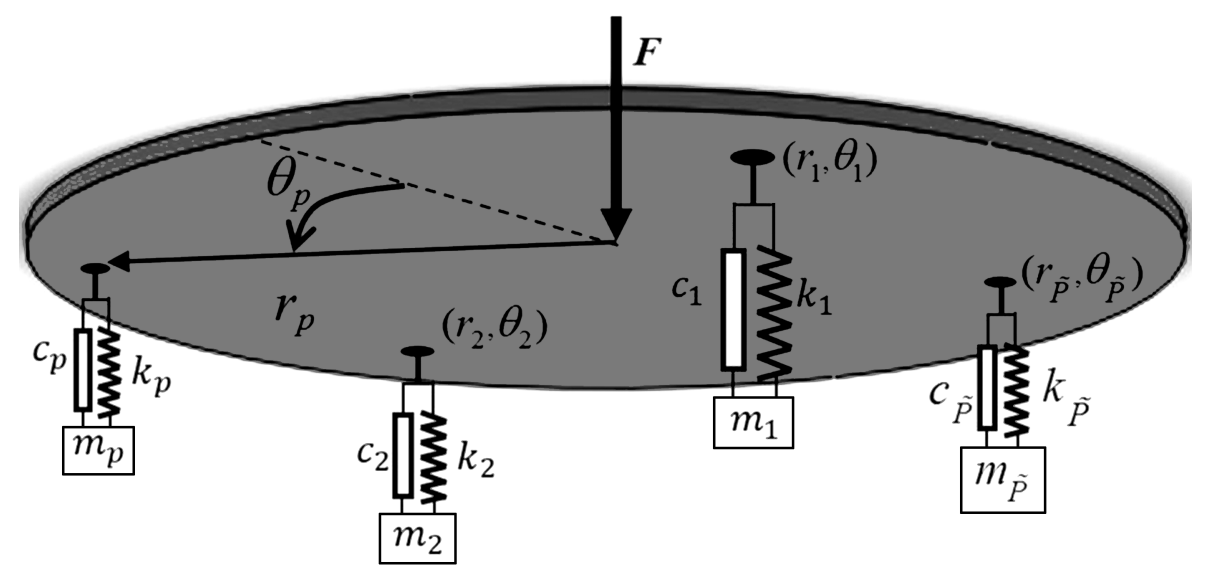

Figure 1. Schematic representation of the circular plate combined with an arbitrary number of Adaptable Vibration Suppressors (AVSs). 


$$
\begin{aligned}
W_{m}(r, \theta)= & {\left[A_{1 m} J_{m}(\beta r)+B_{1 m} I_{m}(\beta r)\right] \sin m \theta } \\
& +\left[A_{2 m} J_{m}(\beta r)+B_{2 m} I_{m}(\beta r)\right] \cos m \theta,
\end{aligned}
$$

$m=0,1,2, \ldots$

in which, the constants should be determined based on the boundary conditions of the plate. It should be mentioned that in Eq. (7), the second-kind Bessel functions, $Y_{m}$ and $K_{m}$ do not appear, because the solution must have finite values in the interior points.

For a free circular plate with radius $R$, bending moment and shear force are zero at free edge $(r=R)$. Applying these boundary conditions, the values of $A_{m}$, $B_{m}$ and $\beta$ will be found. In the absence of suppressors, assume that a harmonic force excites the plate at an arbitrary point $\left(\bar{r}_{0}, \bar{\theta}_{0}\right)$. In this situation, Eq. (5) is modified to:

$$
\begin{aligned}
\ddot{\eta}_{m n}(t) & +2 \zeta_{m n} \omega_{m n} \dot{\eta}_{m n}(t)+\omega_{m n}^{2} \eta_{m n}(t) \\
= & \int_{0}^{2 \pi} \int_{0}^{R} W_{m n}(r, \theta) F_{0}(t) \delta\left(r-\bar{r}_{0}, \theta-\bar{\theta}_{0}\right) r d r d \theta,
\end{aligned}
$$

in which $F_{0}(t)$ is the force acting on the plate. A finite number of suppressors are placed on the plate; name this number $\tilde{P}$. Each suppressor is constituted of mass-spring-damper elements. In the presence of suppressors, Eq. (5) is rewritten as:

$$
\begin{gathered}
\ddot{\eta}_{m n}(t)+2 \zeta_{m n} \omega_{m n} \dot{\eta}_{m n}(t)+\omega_{m n}^{2} \eta_{m n}(t)=\int_{0}^{2 \pi} \int_{0}^{R} \\
W_{m n}(r, \theta) \sum_{p=0}^{\tilde{P}} F_{p}(t) \delta\left(r-\bar{r}_{p}, \theta-\bar{\theta}_{p}\right) r d r d \theta
\end{gathered}
$$

where, $p=1, \ldots, \tilde{P}$ represents each suppressor located at $\left(\bar{r}_{p}, \bar{\theta}_{p}\right)$ and $p=0$ represents the harmonic force located at $\left(\bar{r}_{0}, \bar{\theta}_{0}\right)$. The set of forces exerted by the suppressors can be expressed as:

$$
\begin{aligned}
& F_{p}(t)= k_{p}\left[u_{p}(t)-w\left(\bar{r}_{p}, \bar{\theta}_{p}, t\right)\right] \\
&+c_{p}\left[\dot{u}_{p}(t)-\dot{w}\left(\bar{r}_{p}, \bar{\theta}_{p}, t\right)\right], \\
& p=1,2, . ., \tilde{P}
\end{aligned}
$$

where, $k_{p}, c_{p}, u_{p}$ are stiffness, damping, and displacement of the suppressor, respectively. Substituting Eq. (10) in Eq. (9), the following equation is obtained:

$$
\begin{aligned}
& \ddot{\eta}_{m n}(t)+2 \zeta_{m n} \omega_{m n} \dot{\eta}_{m n}(t) \\
& +\omega_{m n}^{2} \eta_{m n}(t)=F_{0}(t) W_{m n}\left(\bar{r}_{0}, \bar{\theta}_{0}\right) \\
& \quad+\sum_{p=1}^{\tilde{P}}\left(k_{p}\left[u_{p}(t)-w\left(\bar{r}_{p}, \bar{\theta}_{p}, t\right)\right] W_{m n}\left(\bar{r}_{p}, \bar{\theta}_{p}\right)\right. \\
& \left.+c_{p}\left[\dot{u}_{p}(t)-\dot{w}\left(\bar{r}_{p}, \bar{\theta}_{p}, t\right)\right] W_{m n}\left(\bar{r}_{p}, \bar{\theta}_{p}\right)\right) .
\end{aligned}
$$

In this context, $m$ and $n$ are the first and second indices of the mode shapes, where $m=0,1,2, \ldots, M$ and $n=$ $1,2, \ldots, N$. Eq. (11) can be rewritten $(M+1) \times N$ times for all the mode shapes taken into account. In addition to this set of equations used for the plate, the following equation describes the dynamics of each suppressor:

$$
\begin{aligned}
m_{p} \ddot{u}_{p}= & k_{p}\left[w\left(\bar{r}_{p}, \bar{\theta}_{p}, t\right)-u_{p}(t)\right] \\
& +c_{p}\left[\dot{w}\left(\bar{r}_{p}, \bar{\theta}_{p}, t\right)-\dot{u}_{p}(t)\right] .
\end{aligned}
$$

Based on Eqs. (11) and (12), for $(M+1) \times N$ plate modes and $\tilde{P}$ number of suppressors, there are $[(M+$ 1) $\times N]+\tilde{P}$ equations and corresponding number of unknowns. The harmonic excitation force is considered at the center of plate as $\left(\bar{r}_{0}=0, \bar{\theta}_{0}=0\right)$ :

$$
F_{0}(t)=\bar{F} e^{\mathrm{J} \Omega t}
$$

in which $\bar{F}$ and $\Omega$ are the amplitude and frequency of the external force, respectively. Although the excitation is considered to be constituted of only one frequency, the proposed design procedure can be extended to a spectrum of frequencies. Using the method of superposition, the effect of a wide-ranging spectrum can be included. In the case of harmonic forced excitation, the amplitude of coordinates $\eta_{m n}$ and displacement of suppressors $u_{p}$ are described as:

$$
\eta_{m n}(t)=\bar{\eta}_{m n} e^{\mathrm{J} \Omega t}, u_{p}(t)=\bar{u}_{p} e^{\mathrm{J} \Omega t} .
$$

Substituting Eqs. (13) and (14) into Eqs. (11) and (12) and performing some mathematical simplifications and rearrangements, we have:

$$
\begin{aligned}
& \left(-\Omega^{2}+2 \zeta_{m n} \omega_{m n} \Omega \mathrm{J}+\omega_{m n}^{2}\right) \bar{\eta}_{m n} \\
& -\sum_{p=1}^{\tilde{P}}\left(k_{p}+\mathrm{J} \Omega c_{p}\right) \bar{u}_{p} W_{m n}\left(\bar{r}_{p}, \bar{\theta}_{p}\right)+\sum_{p=1}^{\tilde{P}} \\
& \left(k_{p}+\mathrm{J} \Omega c_{p}\right)\left[\sum_{a=0}^{M} \sum_{b=1}^{N} W_{a b}\left(\bar{r}_{p}, \bar{\theta}_{p}\right) \bar{\eta}_{a b}\right] W_{m n}\left(\bar{r}_{p}, \bar{\theta}_{p}\right) \\
& =\bar{F}_{0} W_{m n}\left(\bar{r}_{0}, \bar{\theta}_{0}\right), \\
& \left(-\Omega^{2} m_{p}+\mathrm{J} \Omega c_{p}+k_{p}\right) \bar{u}_{p} \\
& =\left(k_{p}+\mathrm{J} \Omega c_{p}\right)\left[\sum_{a=0}^{M} \sum_{b=1}^{N} W_{a b}\left(\bar{r}_{p}, \bar{\theta}_{p}\right) \bar{\eta}_{a b}\right] .
\end{aligned}
$$

Eq. (15) represents the governing vibration equations of the coupled system under external excitation, including the circular plate and the suppressor. The equations are rearranged in the matrix form of $\Psi \Theta=\Gamma$, in which $\Psi$ is a full-rank matrix of order $[(M+1) \times N]+\tilde{P}, \Theta$ shows a vector which contains the coefficients $\bar{\eta}_{m n}$ and 
$\bar{u}_{p}(m=0,1,2, \ldots, M ; n=1,2, \ldots, N ; p=1,2, \ldots, \tilde{P})$, and $\Gamma$ represents the force vector. Setting the determinant of matrix $\Psi$ equal to zero, the natural frequencies of the coupled system are calculated. Solving the system of equations simultaneously, results in the computation of the generalized coordinates $\bar{\eta}_{m n}$ and the amplitude of displacements $\bar{u}_{p}$.

\section{Devising a complex algorithm with iterative optimization}

A complex optimization algorithm containing several simultaneous sub-algorithms is developed in the MATLAB environment for the hybrid system of circular plate and the AVSs. The frequency of excitation changes over a wide range, while the physical characteristics of the plate and mass value of the suppressors are kept fixed. Vibration suppressors are added to the system one by one and when an AVS is added, its physical characteristics and position are found such that the plate deflection is minimized. The physical parameters and locus of each suppressor are found through a simultaneous search over the possible values. After finding the optimum configuration for the suppressor (i.e., its physical parameters and locus), it is included in the system as a fixed AVS and a similar search procedure begins for the next AVS.

The method for finding the best stiffness and position for each suppressor consists of three major algorithms, which are activated concurrently (as shown in Figure 2 via the dashed box). In each step, the stiffness and position of the suppressor are changed and deflection of the plate is obtained and compared with that of the case with no suppressor. The Root Mean Square (RMS) of plate deflection (at a finite number of points over the plate) is utilized as a comparison criterion for the purpose of optimized design. At each optimization step, the reduction indicator $(\Re)$ is calculated as shown in Box I, which represents the RMS value of plate deflection without suppressors with respect to the case of using suppressors. In this formulation, $D_{r}$ and $D_{\theta}$ are the numbers of interval divisions over the radius and angular position of the circular surface, respectively. The maximum value

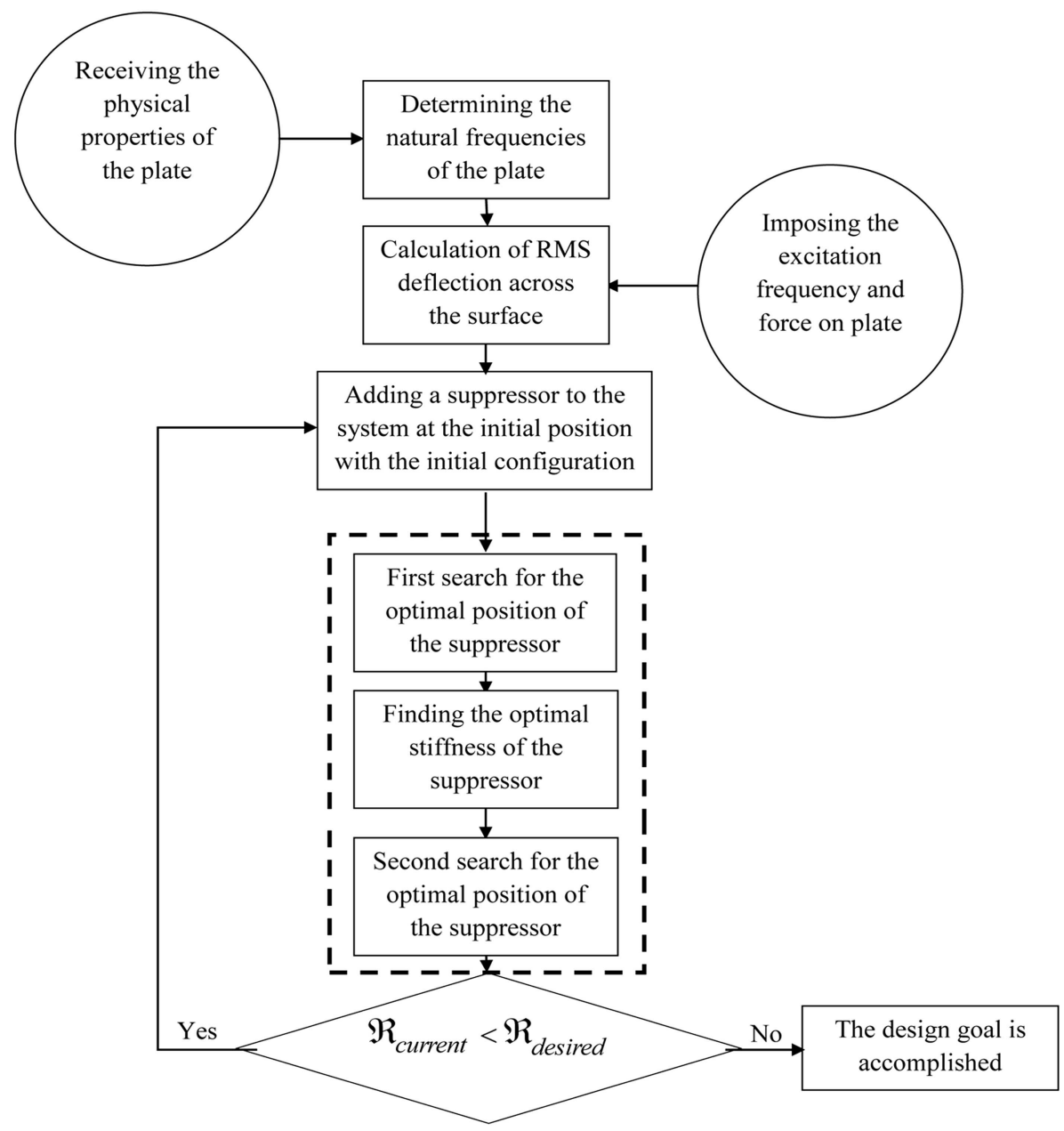

Figure 2. The general method used for obtaining the optimal value of stiffness and position of suppressors. 


$$
\Re=\left(\sum_{i=1}^{D_{r}} \sum_{j=1}^{D_{\theta}} w^{2}\left(r_{i}, \theta_{j}, t\right)\right)_{\text {without absorber }} /\left(\sum_{i=1}^{D_{r}} \sum_{j=1}^{D_{\theta}} w^{2}\left(r_{i}, \theta_{j}, t\right)\right)_{\text {with absorber }}
$$

\section{Box I}

of reduction indicator $\Re_{\max }$ and the corresponding values of stiffness and position represent the optimum configuration of the suppressor.

The general procedure of the algorithm is presented in Figure 2. Each new suppressor is first placed at the central point with an initial stiffness equal to the mass of suppressor multiplied by the square of the excitation frequency, $\Omega$ (i.e. $k_{\text {initial }}=m \Omega^{2}$ ). With the stiffness fixed, the best position for the suppressor is found. Fixing the suppressor in that position, a search for finding the optimal stiffness value is carried out. For the second time, the stiffness is fixed and a second search for the best position is conducted. The algorithm for finding the best value of stiffness and best locus will be discussed next. Through these three steps, the optimum framework for the suppressors, which corresponds to the maximum possible value of reduction indicator, is determined.

Step 1. To find the optimal locus of the suppressors, each new suppressor is initially placed at the central point. The suppressor is placed in 100 separate points, which are the intersections of 10 equally spaced circles and 10 equally spaced diametric lines (as shown in Figure 3 by "Search Domain"). The point corresponding to the maximum value of reduction indicator is found and used for the rest of the search procedure. The method used to determine the optimal locus of the suppressors is presented in Figure 3.

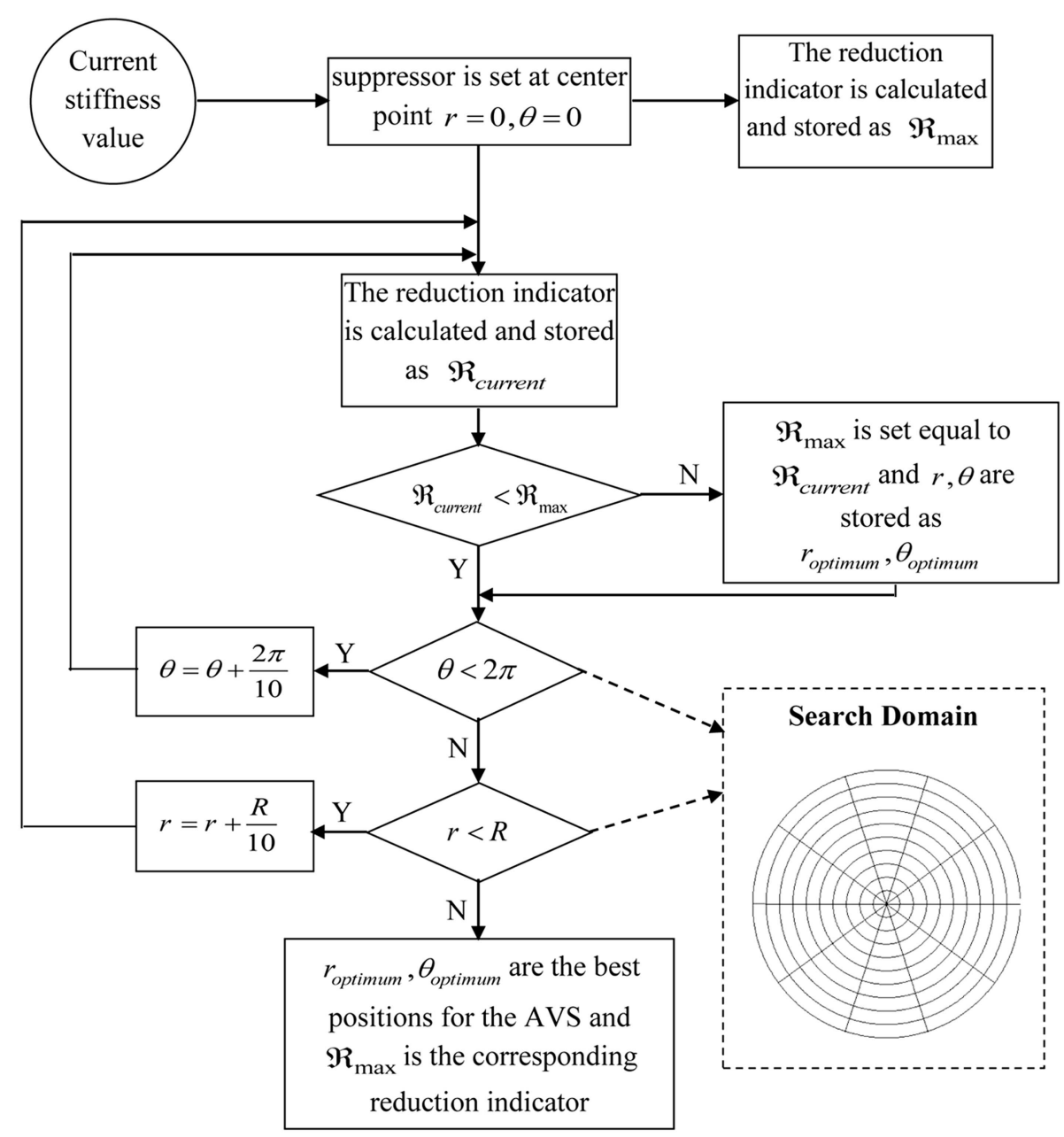

Figure 3. The method used for obtaining the optimum locus of the suppressors. 
Step 2. In the next step, the suppressor is fixed in the best position and a "rough search" over the optimum value of stiffness begins. Stiffness of the suppressor is increased gradually from zero to a value that covers the considered highest natural frequency of the plate. The stiffness corresponding to the highest value of reduction indicator represents the approximate value of optimal stiffness. A second and more precise search, called "fine search," is conducted to find the exact value of optimal stiffness. In comparison with "rough search," here, the search for stiffness value $k$ is performed with a much smaller resolution. The procedure followed to determine the optimum values of stiffness of the suppressor through rough/fine searches is presented in Figure 4.
Step 3. Finding the best value of stiffness, a second procedure similar to the initial one is conducted to reassure that the first found positions represent the optimal ones. As soon as the optimum locus and stiffness of a suppressor are found, the suppressor is set on the system with the optimum configurations, and the search begins for optimizing the next suppressor. This procedure is repeated until the reduction indicator of the hybrid system reaches a desired value, called $\Re_{\text {desired }}$. This desired value is selected such that an acceptable large reduction is obtained while the number of suppressors is kept low to the possible extent (to prevent complex design). This compromised selection of $\Re_{\text {desired }}$ is explained more in the next results.

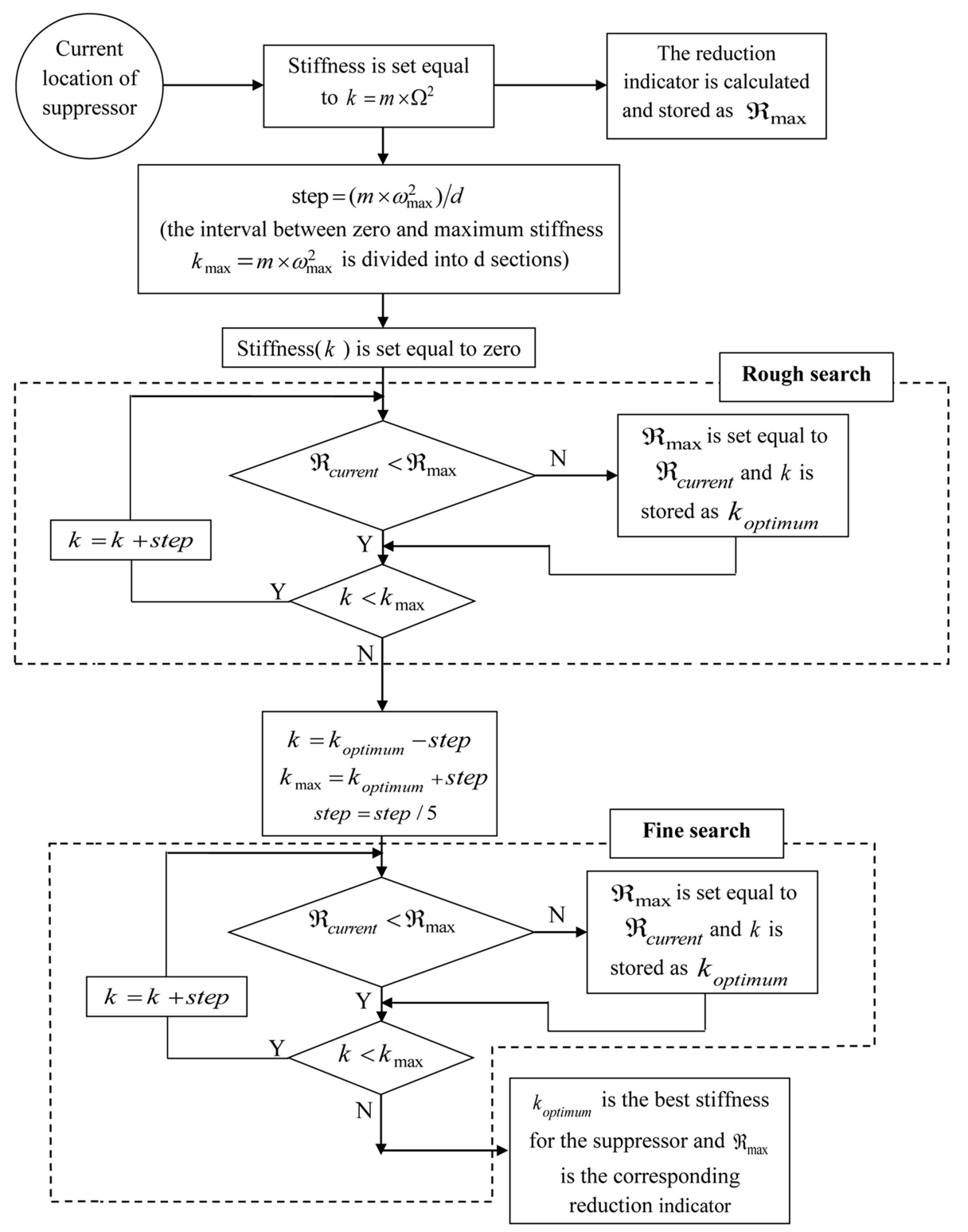

Figure 4. The method used for obtaining the optimum stiffness value for each suppressor, including 'rough' and 'fine' searches. 


\section{Simulations for process optimization (results and discussion)}

\subsection{Physical characteristics of the plate}

In this paper, an Aluminum circular plate is considered. As already discussed, the harmonic excitation is applied at the central point with a uniform amplitude of $\bar{F}=1 \mathrm{kN}$ and initial frequency of $\Omega=20 \mathrm{rad} / \mathrm{s} \approx 3.18 \mathrm{~Hz}$ (this is the initial value of excitation frequency, which can vary as presented next). Also, the next results can be presented in a similar way for other values of force amplitude and location.

The devised algorithm for determining the optimal configuration of Adaptable Vibration Suppressors (AVSs) is established in a general user-friendly program that addresses the needs of the user. In order to simplify the proposed relations of the previous section, some model parameters are set constant while the other properties vary in a wide spectrum. Among them, plate radius $(R=4 \mathrm{~m})$, mass per unit area $(\rho=$ $\left.13.5 \mathrm{~kg} / \mathrm{m}^{2}\right)$, damping coefficient $(\zeta=0.2)$, Young's modulus $(E=69 \mathrm{GPa})$, Poisson's ratio $(\nu=0.33)$, and suppressor mass $(m=10 \mathrm{~kg})$ are kept constant. Nine possible modes are considered for the circular plate (i.e., $m=0,1, \ldots, M, n=1,2, \ldots, N, M=2, N=3$ ) and the plate is divided into 100 elements, consisting of 10 circular sections and 10 diametric sections $\left(D_{r}=10\right.$, $\left.D_{\theta}=10\right)$. General physical properties of the plate and suppressors are exhibited in able 1 . The first seven mode shapes of the plate are represented in Figure 5, while its natural frequencies are given in Table 2 .

\subsection{Design of optimal AVSs and their effect on attenuating undesirable vibrations}

The algorithm that was discussed in Section 3 is utilized for finding the optimal configuration of Adaptable Vibration Suppressors (AVSs) that are mounted on the circular plate in 5 different states of resonance and non-resonance excitations. In the resonance cases, the excitation frequency approaches one of the natural frequencies of the plate $\left(\Omega=\omega_{21}, \omega_{02}, \omega_{12}, \omega_{22}\right)$. These are the first 4 natural frequencies that are sorted by magnitude. Moreover, one arbitrary excitation frequency that is located between the first and second natural frequencies is selected as a non-resonance case $\left(\omega_{21}<\Omega=20 \mathrm{rad} / \mathrm{s}<\omega_{02}\right)$. The results corresponding to this non-resonance case are presented while similar results can be derived for other non-resonance excitations.

4.2.1. Optimum number of AVSs to place on the plate Placing the suppressors on the plate follows a stepby-step procedure in which a new suppressor is placed in the system such that it minimizes the RMS value of deflection. To investigate the effect of adding suppressors, their number varies gradually from 1 to 30 (i.e., $p=1,2, \ldots, 30, \tilde{P}_{\max }=30$ ). At each iteration of adding a new suppressor, the reduction indicator $(\Re)$ is found. This procedure is repeated for various resonance/non-resonance conditions, as shown in Figure 6. It should be noticed that, to present a continuous plot in Figure 6 and similar next plots, a linear interpolation is used for the $\Re$ variation versus the number of AVSs. As it is observed and expected,

Table 1. Physical characteristics of the plate, attached vibration suppressors and central harmonic excitation.

\begin{tabular}{llll}
\hline \multicolumn{2}{c}{ Plate specifications } & \multicolumn{2}{c}{ Excitation specifications } \\
\hline Mass per unit area & $\rho=13.5 \mathrm{kgm}^{2}$ & Amplitude of excitation & $\bar{F}=1 \mathrm{kN}$ \\
Radius & $R=4 \mathrm{~m}$ & Frequency of excitation & $\Omega=20 \mathrm{rad} / \mathrm{s}$ \\
\cline { 2 - 4 } Thickness & $h=5 \mathrm{~mm}$ & \multicolumn{2}{c}{ Initial suppressors' specifications } \\
\cline { 3 - 4 } Young's modulus & $E=69 \mathrm{GPa}$ & Mass of each suppressor & $m=10 \mathrm{~kg}$ \\
Poisson's ratio & $\nu=0.33$ & Initial stiffness of each suppressor & $k=4 \mathrm{kN} / \mathrm{m}$ \\
Damping coefficient & $\zeta=0.2$ & Initial location of each suppressor & $r=0, \theta=0$ \\
\hline
\end{tabular}

Table 2. Natural frequencies of the plate in the absence of suppressors.

\begin{tabular}{|c|c|c|c|c|}
\hline & & \multicolumn{3}{|c|}{ 2nd mode index } \\
\hline \multicolumn{2}{|c|}{$\omega_{m n}(\mathrm{rad} / \mathrm{s})$} & $n=1$ & $n=2$ & $n=3$ \\
\hline \multirow{3}{*}{ 1st mode index } & $m=0$ & & $\omega_{02}=24.82$ & $\omega_{03}=105.35$ \\
\hline & $m=1$ & & $\omega_{12}=56.08$ & $\omega_{13}=163.58$ \\
\hline & $m=2$ & $\omega_{21}=14.35$ & $\omega_{22}=96.33$ & $\omega_{23}=229.27$ \\
\hline
\end{tabular}




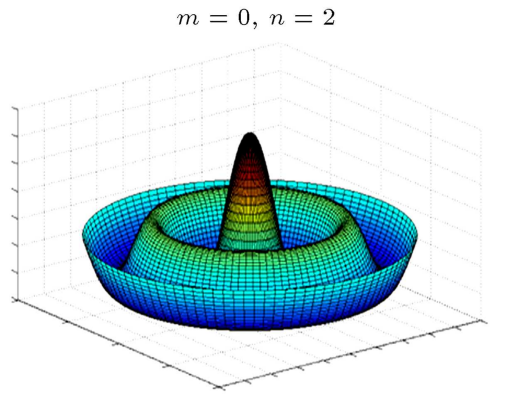

(a)

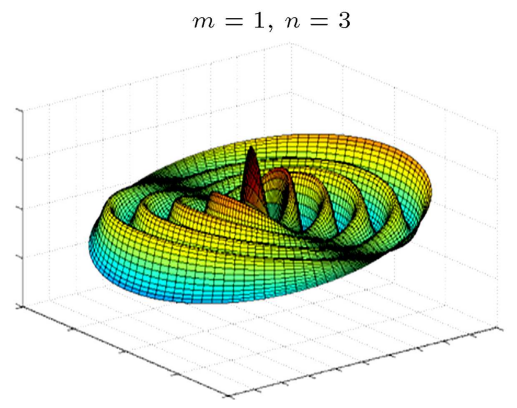

(d)

$m=2, n=1$

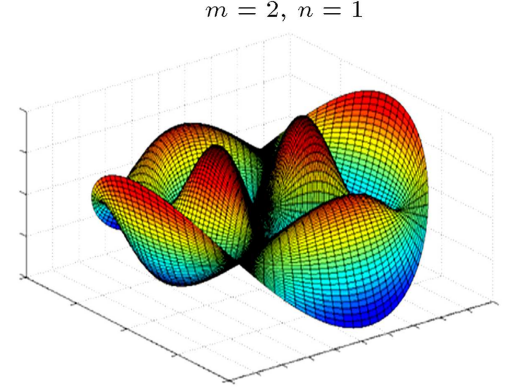

(e)

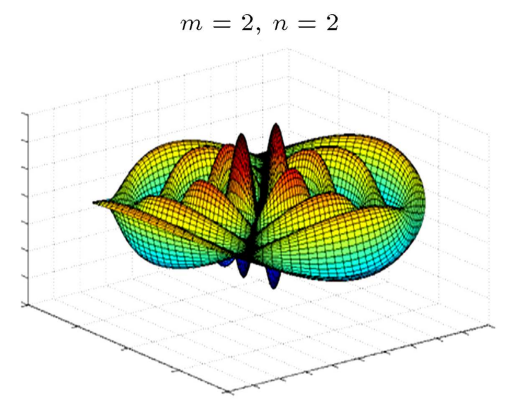

(f)

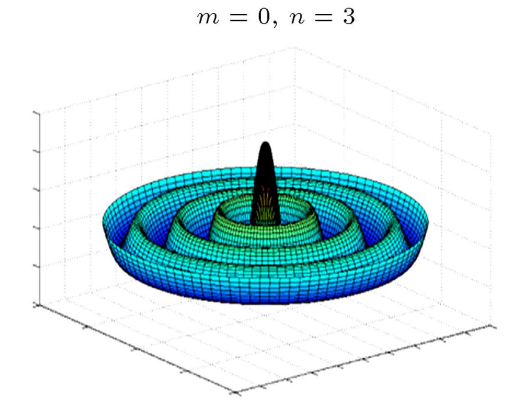

(b)

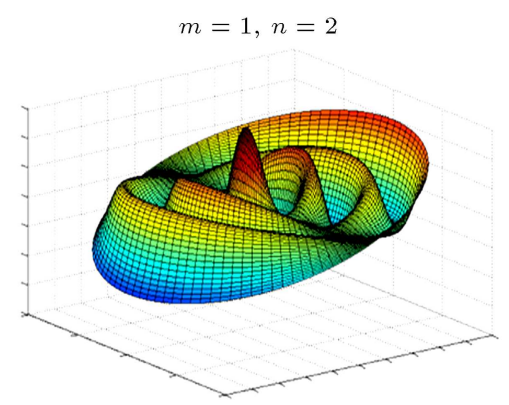

(c)

$m=2, n=3$

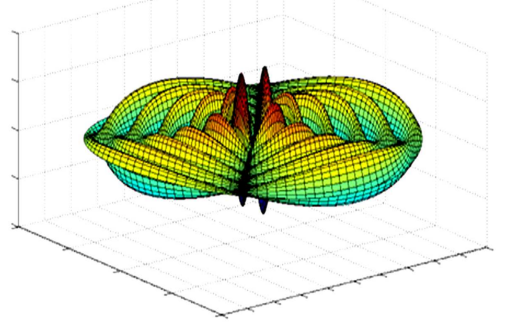

(g)

Figure 5. The first seven natural mode shapes of the plate; each row represents the first mode shape number $(m)$, and each column represents the second mode shape number $(n)$.

Table 3. Values of reduction indicator $(\Re)$ when 5 AVSs are attached gradually $(p=1,2, \ldots, 5)$ under various resonance and non-resonance excitation frequencies.

\begin{tabular}{ccccccc}
\hline $\begin{array}{c}\text { Number of } \\
\text { suppressors }\end{array}$ & $\begin{array}{c}\text { Reduction } \\
\text { indicator }\end{array}$ & $\boldsymbol{\Omega} \approx \boldsymbol{\omega}_{\mathbf{2}} \mathbf{1}$ & $\boldsymbol{\Omega} \approx \boldsymbol{\omega}_{\mathbf{0} \mathbf{2}}$ & $\boldsymbol{\Omega} \approx \boldsymbol{\omega}_{\mathbf{1} \mathbf{2}}$ & $\boldsymbol{\Omega} \approx \boldsymbol{\omega}_{\mathbf{2} \mathbf{2}}$ & $\boldsymbol{\omega}_{\mathbf{2} \mathbf{1}}<\boldsymbol{\Omega}<\boldsymbol{\omega}_{\mathbf{0 2}}$ \\
\hline$\tilde{P}=1$ & $\Re_{1}$ & 27.51 & 54.44 & 4.08 & 6.61 & 47.30 \\
$\tilde{P}=2$ & $\Re_{2}$ & 46.17 & 79.38 & 7.49 & 15.96 & 69.54 \\
$\tilde{P}=3$ & $\Re_{3}$ & 58.17 & 241.20 & 11.42 & 20.42 & 177.20 \\
$\tilde{P}=4$ & $\Re_{4}$ & 131.30 & 325.60 & 17.14 & 24.23 & 253.80 \\
$\tilde{P}=5$ & $\Re_{5}$ & 132.80 & 341.60 & 19.77 & 29.05 & 275.80 \\
\hline
\end{tabular}

introducing more suppressors to the system increases the reduction indicator value. Also, high reduction values are observed for various cases of resonance/nonresonance conditions.

Based on these results, a specific number of suppressors to place on the system is chosen and used for the rest of the studies. Comparing the plots in Figure 6 reveals that using five $\operatorname{AVSs}(\tilde{P}=5)$ on the system can result in a reasonably high value of reduction indicator. On the other hand, with $\tilde{P}=$ 5 , a relatively low number of suppressors are used, which make the design more practical in industry (in comparison with, e.g., $\tilde{P}=10$ or higher). Values of reduction indicator based on the number of suppressors placed in the system for the first five attached AVSs under various excitations are presented in Table 3 . 


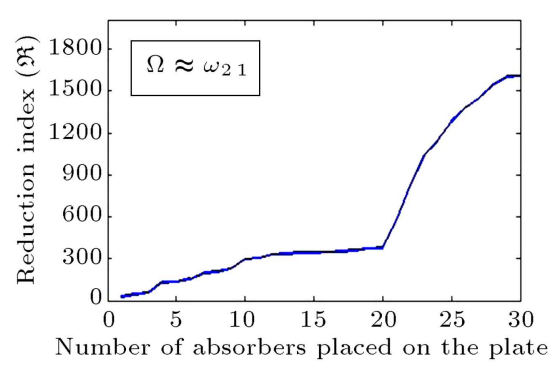

(a)

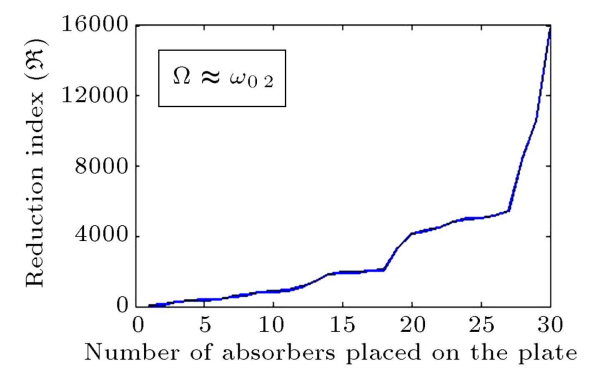

(b)

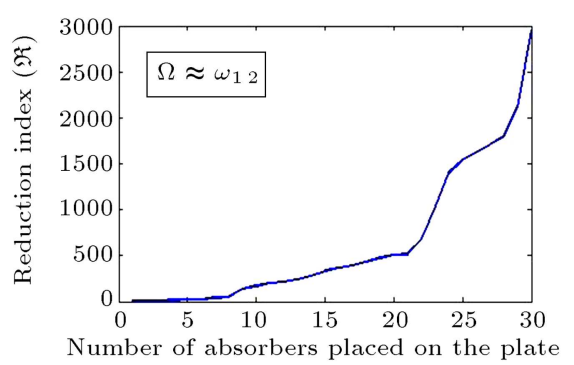

(c)

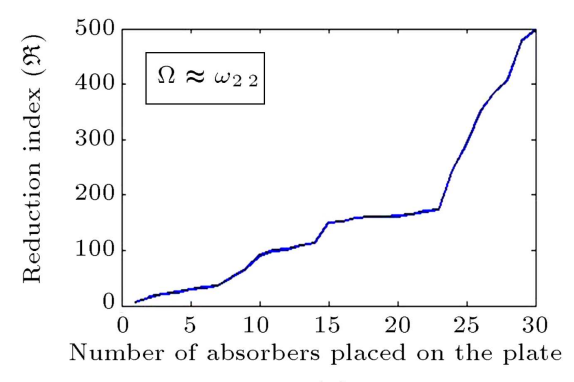

(d)

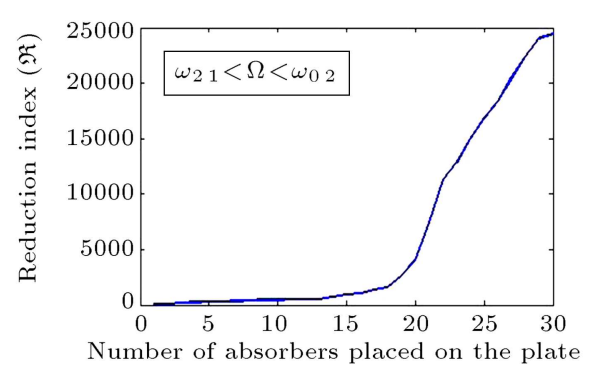

(e)

Figure 6. The amount of reduction indicator $(\Re)$ for various resonance/non-resonance conditions under the effect of different numbers of AVSs placed on the plate: (a) $\Omega \approx \omega_{21}$, (b) $\Omega \approx \omega_{02}$, (c) $\Omega \approx \omega_{12}$, (d) $\Omega \approx \omega_{22}$, and (e) $\omega_{21}<\Omega<\omega_{02}$.

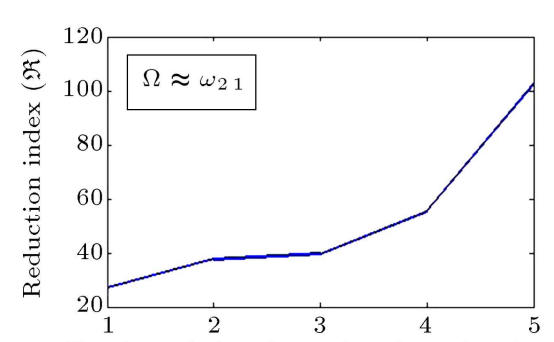

Number of absorbers placed on the plate

(a)

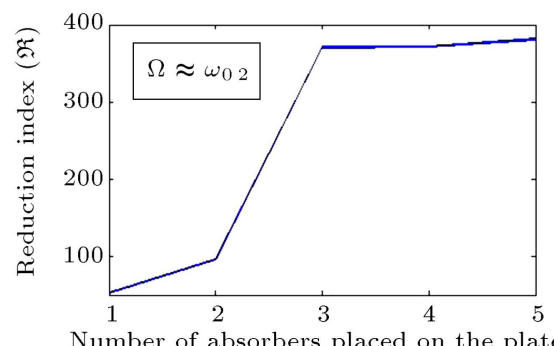

Number of absorbers placed on the plate

(b),

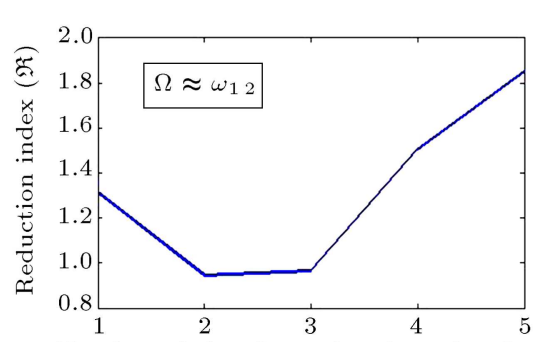

Number of absorbers placed on the plate

(c)

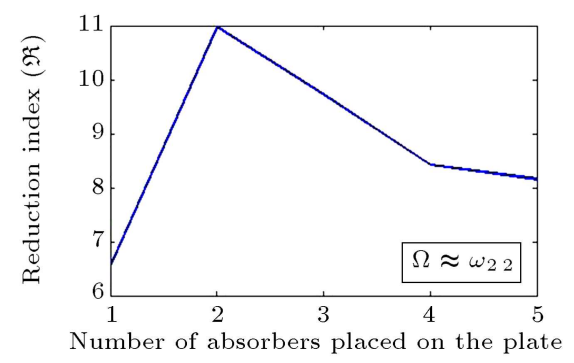

(d)

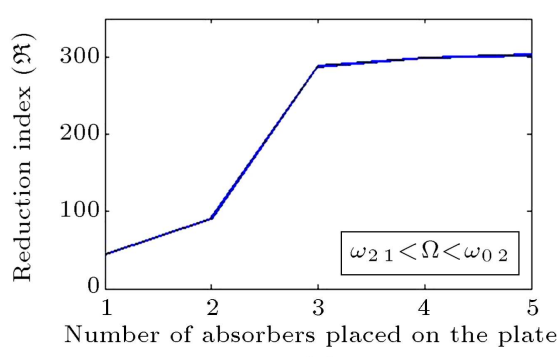

(e)

Figure 7. The amount of reduction indicator $(\Re)$ when five identical AVSs are used under different resonance and non-resonance conditions: (a) $\Omega \approx \omega_{21}$, (b) $\Omega \approx \omega_{0,2}$, (c) $\Omega \approx \omega_{12}$, (d) $\Omega \approx \omega_{22}$, and (e) $\omega_{21}<\Omega<\omega_{02}$.

\subsubsection{The effect of using identical AVSs on vibration suppression}

To achieve a simple design, identical suppressors can be used. In the previously demonstrated algorithm, each AVS has its specific stiffness and position. In this section, definite number of similar AVSs are placed on the plate, while the search for the best positions has already been conducted. The used stiffness value is equal to the arithmetic mean of stiffness of the AVSs found in Section 4.2.1. For instance, this procedure is carried out for two cases of 5 and 30 AVSs.

Figures 7 and 8 represent the reduction indicator $(\Re)$ based on the maximum number of suppressors placed on the plate for two cases of 5 and 30 suppressors, respectively. For instance, Figure 7(a) demonstrates the case when excitation frequency approaches 


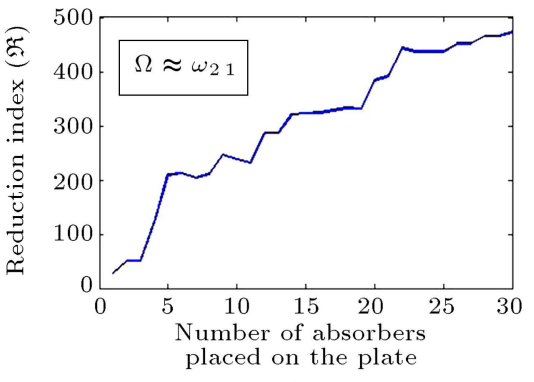

(a)

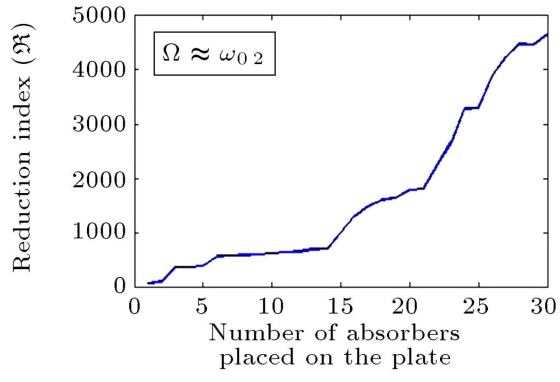

(b)

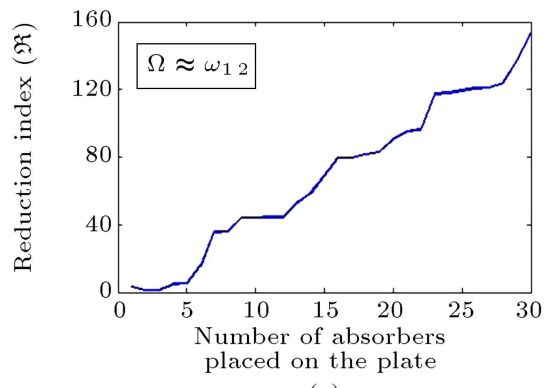

(c)

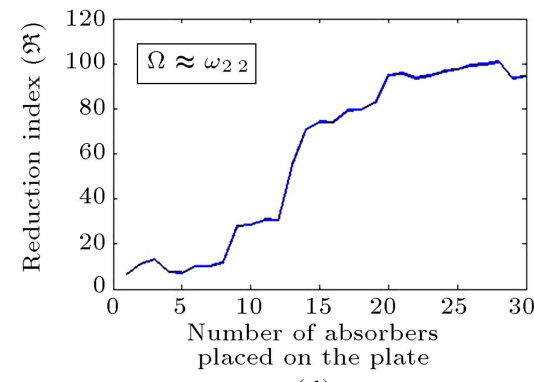

(d)

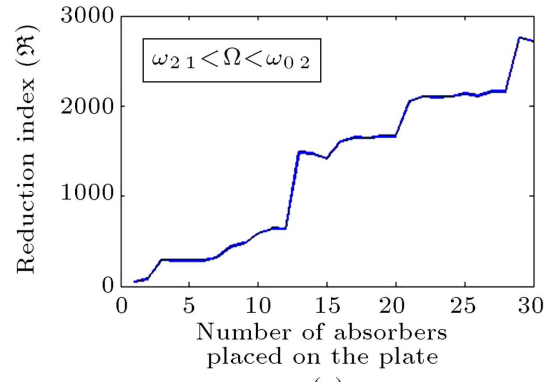

(e)

Figure 8. The amount of reduction indicator $(\Re)$ when 30 identical AVSs are used under different resonance and non-resonance conditions: (a) $\Omega \approx \omega_{21}$, (b) $\Omega \approx \omega_{02}$, (c) $\Omega \approx \omega_{12}$, (d) $\Omega \approx \omega_{22}$, and (e) $\omega_{21}<\Omega<\omega_{02}$.

$\omega_{2} 1$ and the stiffness values of all 5 suppressors are equal to the arithmetic mean of stiffness of the suppressors found in Figure 6(a). According to Figure 7(a), using 5 identical AVSs results in $\Re \approx 100$. On the other hand, according to Figure $6($ a) or Table 3 , for $\tilde{P}=5$ and $\Omega=\omega_{2}, \Re \approx 132.80$ is obtained, where each suppressor has its own optimum value of stiffness. Therefore, using the identical AVSs may lead to smaller amounts of reduction.

\subsubsection{The effect of optimized AVSs on time responses and plate deflection}

As already discussed, using five suppressors leads to large values of reduction indicator while the system design is kept simple (in comparison with high numbers of AVSs). Therefore, in the next simulation results, it is assumed that 5 AVSs are implemented. Using the proposed optimization algorithm, optimum values for the position and stiffness of the AVSs for different resonance and non-resonance cases are found, as detailed in Table 4 and presented graphically in Figure 9.

In order to analyze the impact of the vibration suppressors on the time response of the plate, the point that experiences the maximum oscillation amplitude is found for various cases including two states with suppressors and without suppressors. The point corresponding to maximum amplitude is named 'maxpoint.' Time history of 'max-point' for various resonance/non-resonance conditions, with/without optimum AVSs, is shown in Figure 10. Time response is presented for the time domain of $0<t<5 \times(2 \pi / \Omega)$. As it is observed, AVSs act effectively in attenuating the plate vibrations, especially under resonance conditions.

Considering the previously found results in Figure 10 (left column), the critical time corresponding to maximum deflection of 'max-point' in the case of no suppressors is found for each condition and named $t^{*}$. Figure 11 shows the deflection of the plate at this critical time $\left(t^{*}\right)$ with/without optimum AVSs. According to Figure 11 and under resonance/nonresonance conditions, implementation of AVSs leads to the attenuation of plate deflection.

\subsubsection{Examination of the efficiency of optimum AVSs via frequency response analysis}

To study the effectiveness of AVSs design for an extensive range of excitation frequencies, harmonic excitation frequency is assumed to vary within $0<$ $\Omega<250 \mathrm{rad} / \mathrm{s}$ such that it covers the first seven natural frequencies of the plate. Figure 12 shows the reduction indicator value $\Re$ (RMS value of plate deflection without suppressor with respect to the case with optimum AVSs) for a broad spectrum of excitation frequencies. It should be noticed that in each plot of Figure 12, a set of optimum suppressors, designed for each case of resonance and non-resonance conditions, is used. For instance, the set of optimum AVSs designed for the first resonance condition (i.e., 5 AVSs with specifications given in Table 4 , with $\Omega \approx \omega_{21}$ ) is used for the range of $0<\Omega<250 \mathrm{rad} / \mathrm{s}$ and its corresponding reduction indicator $\Re$ is shown in Figure 12(a).

According to Figure 12, large reductions can be obtained over an extensive range of excitation 


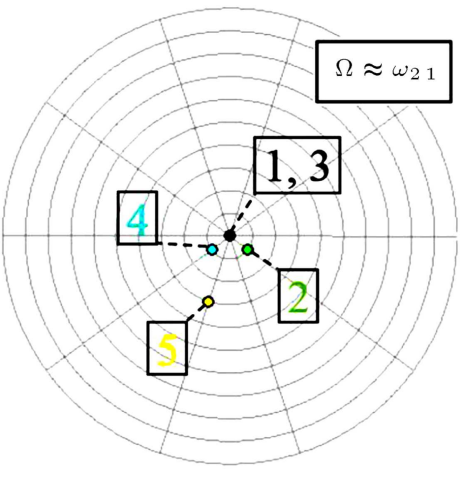

(a)

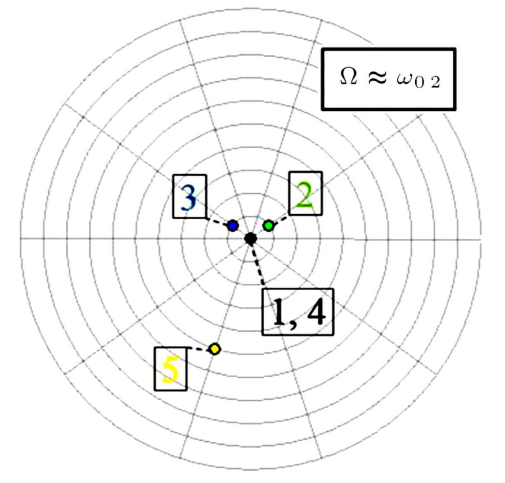

(b)

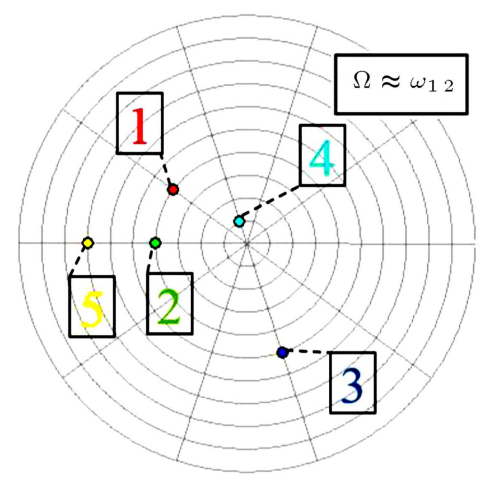

(c)

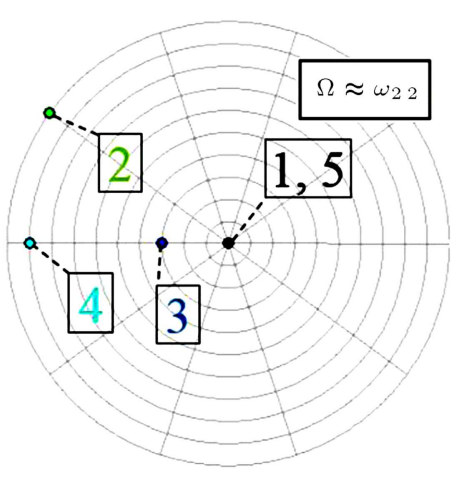

(d)

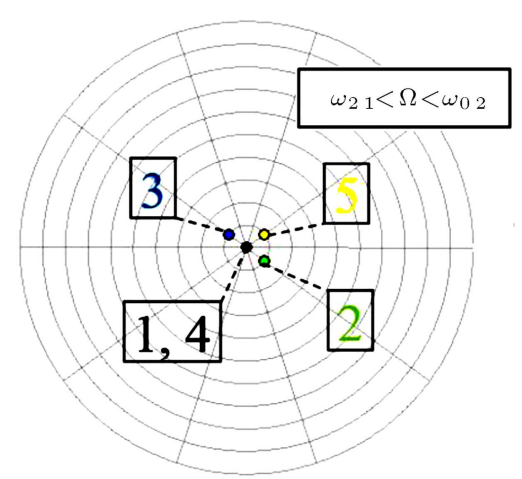

(e)

Figure 9. Optimum positions of the first 5 -AVSs under different resonance and non-resonance conditions: (a) $\Omega \approx \omega_{21}$, (b) $\Omega \approx \omega_{02},\left(\right.$ c) $\Omega \approx \omega_{12},\left(\right.$ d) $\Omega \approx \omega_{22}$, and (e) $\omega_{21}<\Omega<\omega_{02}$.

Table 4. Optimum values for the position, stiffness, and mass of AVSs for different resonance/non-resonance cases.

\begin{tabular}{|c|c|c|c|c|c|c|}
\hline Case & Property & $\begin{array}{c}\text { 1st } \\
\text { suppressor }\end{array}$ & $\begin{array}{c}\text { 2nd } \\
\text { suppressor }\end{array}$ & $\begin{array}{c}\text { 3rd } \\
\text { suppressor }\end{array}$ & $\begin{array}{c}\text { 4th } \\
\text { suppressor }\end{array}$ & $\begin{array}{c}\text { 5th } \\
\text { suppressor }\end{array}$ \\
\hline \multirow{4}{*}{$\Omega \approx \omega_{21}$} & Radius (m) & 0.0 & 0.4 & 0.0 & 0.4 & 1.2 \\
\hline & Angle (rad) & 0.0 & 5.65 & 0.0 & 3.76 & 4.39 \\
\hline & Stiffness $(\mathrm{kN} / \mathrm{m})$ & 1.70 & 1.59 & 1.65 & 1.65 & 2.08 \\
\hline & Mass (kg) & 10.0 & 10.0 & 10.0 & 10.0 & 10.0 \\
\hline \multirow{4}{*}{$\Omega \approx \omega_{02}$} & Radius (m) & 0.0 & 0.4 & 0.4 & 0.0 & 2.0 \\
\hline & Angle (rad) & 0.0 & 0.62 & 2.51 & 0.0 & 4.39 \\
\hline & Stiffness $(\mathrm{kN} / \mathrm{m})$ & 5.29 & 4.51 & 4.51 & 4.91 & 5.11 \\
\hline & Mass (kg) & 10.0 & 10.0 & 10.0 & 10.0 & 10.0 \\
\hline \multirow{4}{*}{$\Omega \approx \omega_{12}$} & Radius (m) & 1.6 & 1.6 & 2.0 & 0.4 & 2.8 \\
\hline & Angle (rad) & 2.51 & 3.14 & 5.02 & 1.88 & 3.14 \\
\hline & Stiffness $(\mathrm{kN} / \mathrm{m})$ & 26.27 & 24.56 & 25.12 & 12.37 & 18.49 \\
\hline & Mass (kg) & 10.0 & 10.0 & 10.0 & 10.0 & 10.0 \\
\hline \multirow{4}{*}{$\Omega \approx \omega_{22}$} & Radius (m) & 0.0 & 4.0 & 1.2 & 3.6 & 0.0 \\
\hline & Angle (rad) & 0.0 & 2.51 & 3.14 & 3.14 & 0.0 \\
\hline & Stiffness $(\mathrm{kN} / \mathrm{m})$ & 401.07 & 59.33 & 68.66 & 76.47 & 106.94 \\
\hline & Mass (kg) & 10.0 & 10.0 & 10.0 & 10.0 & 10.0 \\
\hline \multirow{4}{*}{$\omega_{21}<\Omega<\omega_{02}$} & Radius (m) & 0.0 & 0.4 & 0.4 & 0.0 & 0.4 \\
\hline & Angle (rad) & 0.0 & 5.65 & 2.51 & 0.0 & 0.62 \\
\hline & Stiffness $(\mathrm{kN} / \mathrm{m})$ & 4.19 & 3.67 & 3.55 & 3.94 & 3.77 \\
\hline & Mass (kg) & 10.0 & 10.0 & 10.0 & 10.0 & 10.0 \\
\hline
\end{tabular}



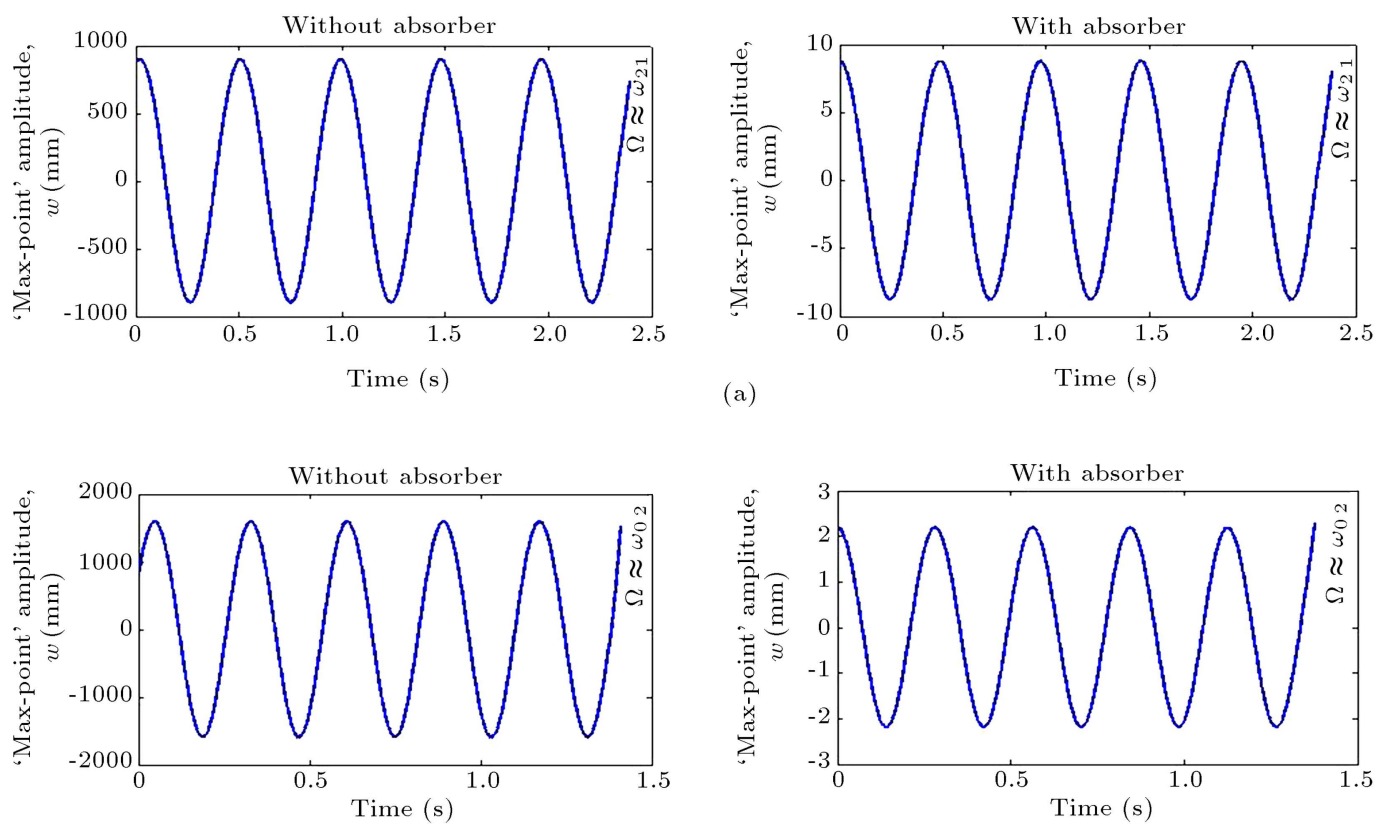

(b)
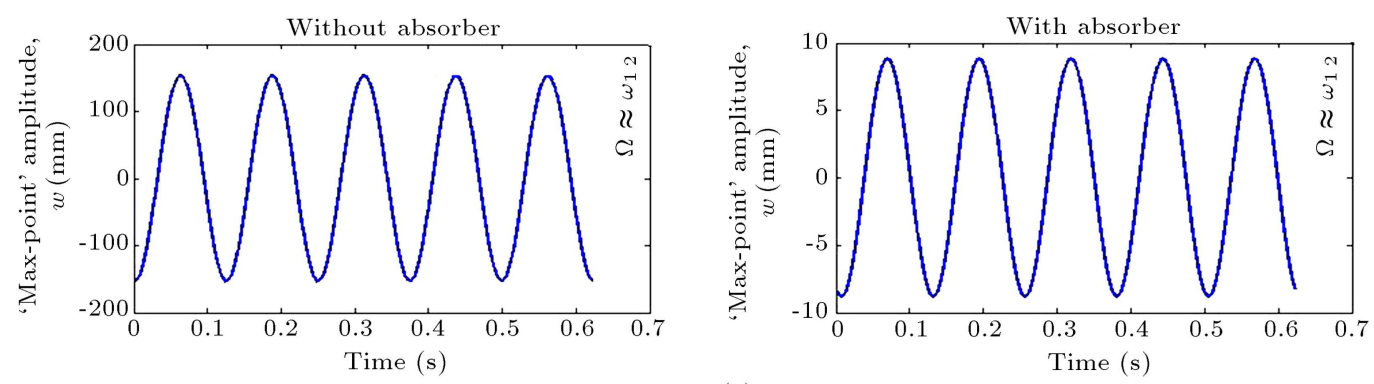

(c)
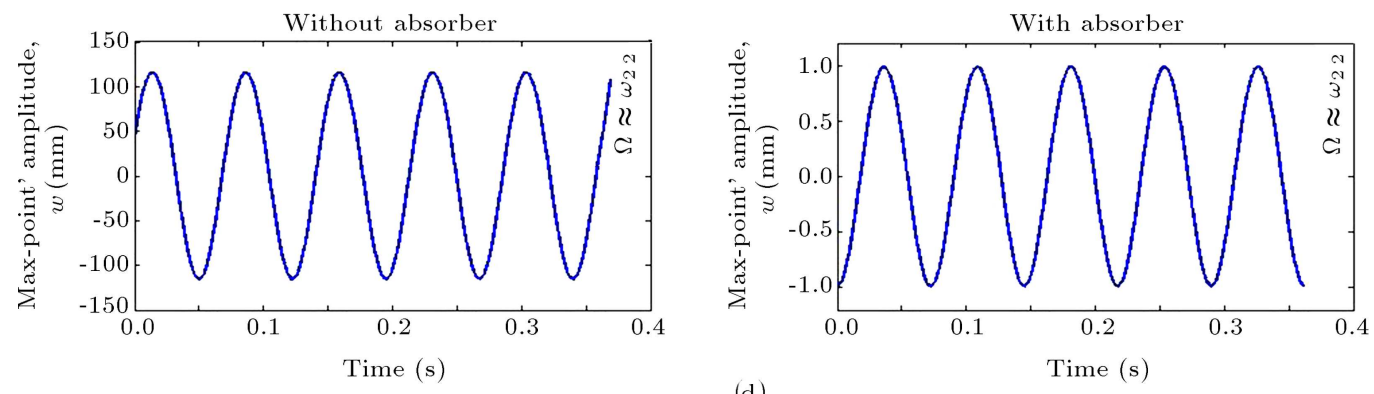

(d)
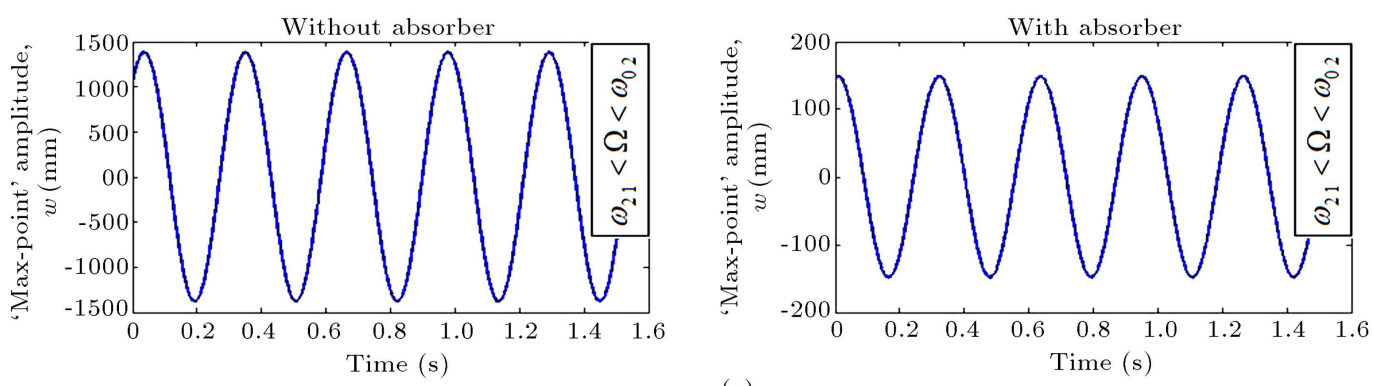

(e)

Figure 10. Response of the 'max-point' vibration amplitude in different resonance/non-resonance conditions in the time domain: (a) $\Omega \approx \omega_{21}$, (b) $\Omega \approx \omega_{02}$, (c) $\Omega \approx \omega_{12}$, (d) $\Omega \approx \omega_{22}$, and (e) $\omega_{21}<\Omega<\omega_{02}$; without (left column) and with (right column) optimum AVSs. 

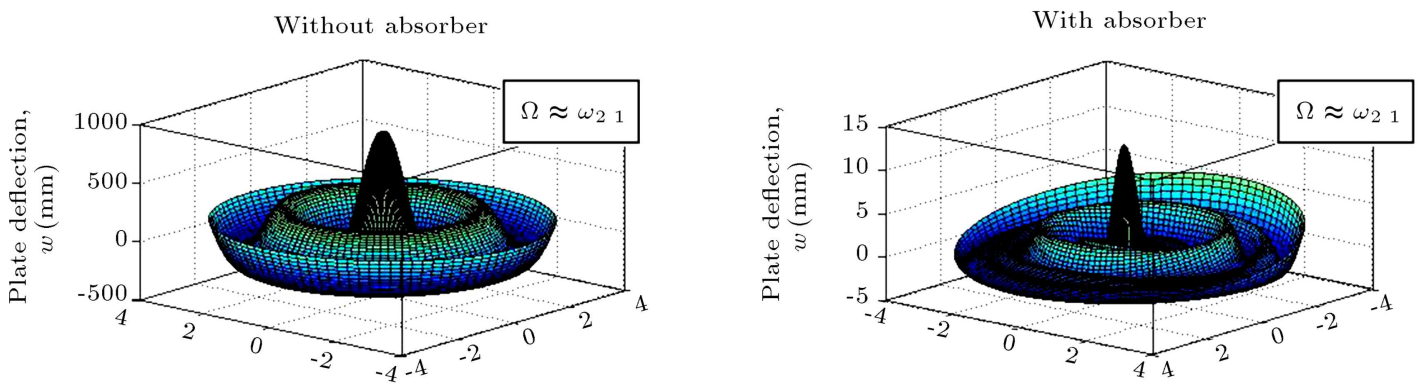

(a)
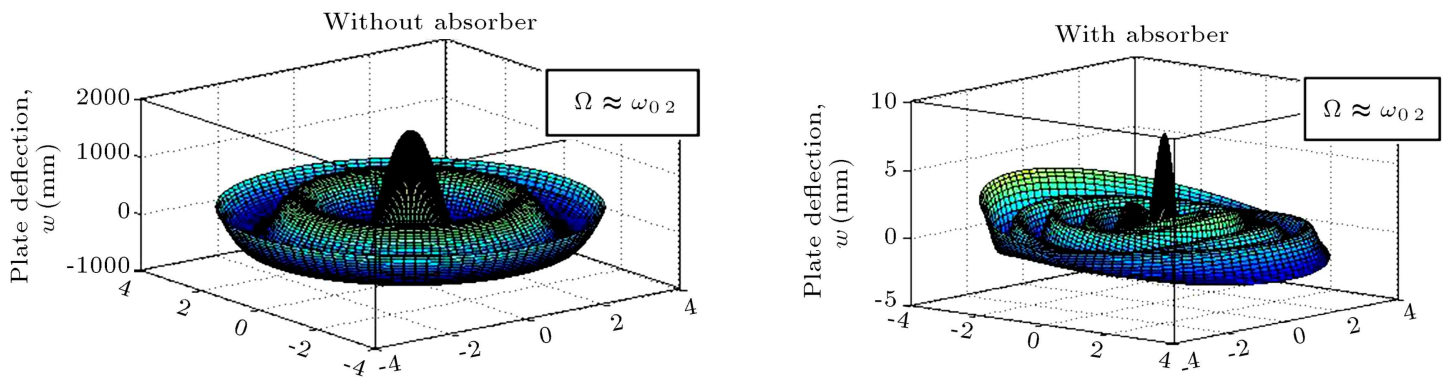

(b)
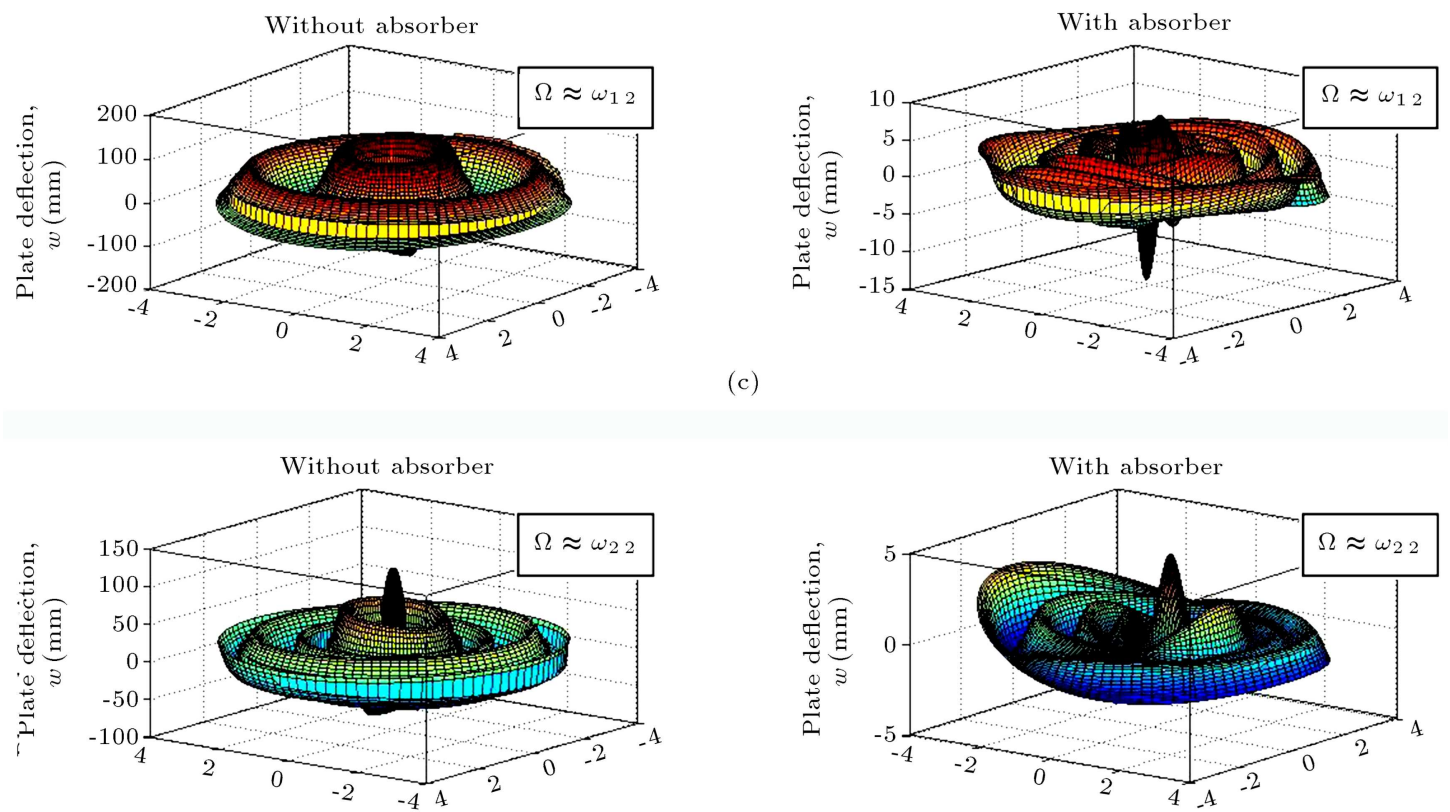

(d)
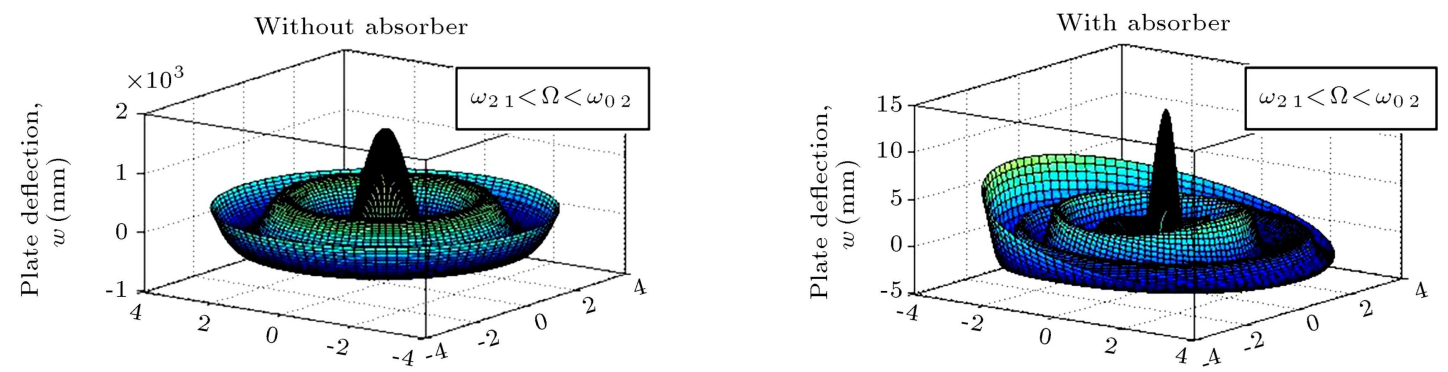

(e)

Figure 11. Plate deflection (at the critical time $t^{*}$ ) in various resonance/non-resonance cases: (a) $\Omega \approx \omega_{21}$, (b) $\Omega \approx \omega_{02}$, (c) $\Omega \approx \omega_{12}$, (d) $\Omega \approx \omega_{22}$, and (e) $\omega_{21}<\Omega<\omega_{02}$; without (left column) and with (right column) optimum AVSs. 


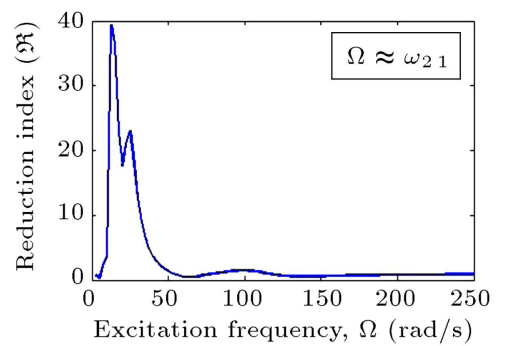

(a)

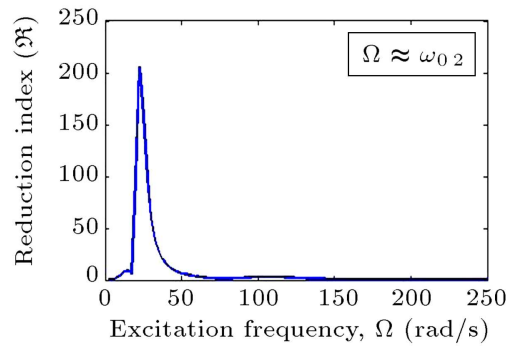

(b)

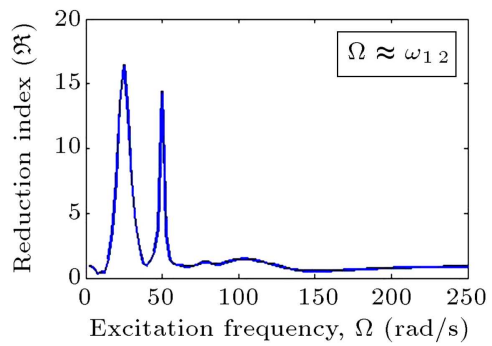

(c)

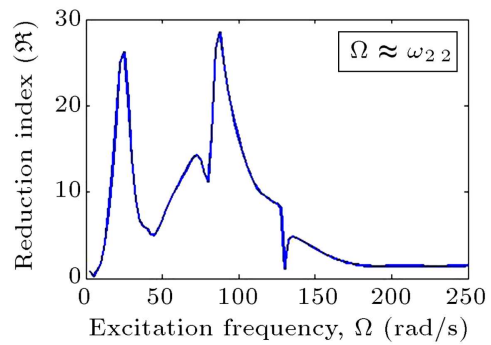

(d)

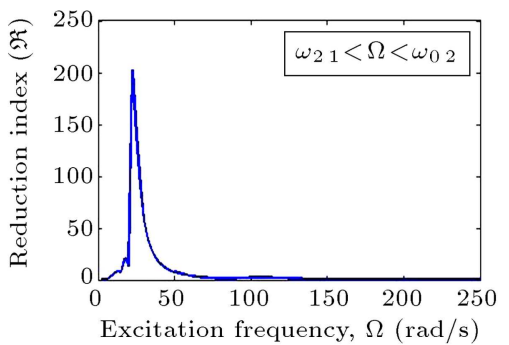

(e)

Figure 12. The indicator of the amount of reduction $(\Re)$ over a broad spectrum of excitation frequencies, while the AVSs designed for (a) $\Omega \approx \omega_{21}$, (b) $\Omega \approx \omega_{02}$, (c) $\Omega \approx \omega_{12}$, (d) $\Omega \approx \omega_{22}$, and (e) $\omega_{21}<\Omega<\omega_{02}$ are utilized.

frequencies in the presence of optimum AVSs. Also, it is observed that vibration suppression is most evident in the vicinity of excitation frequencies that correspond to the resonance cases. For instance, in Figure $12(\mathrm{a})$ where the utilized AVSs are designed for $\Omega=\omega_{21}$, maximum reduction is portrayed around $\Omega=\omega_{21} \approx$ $14.35 \mathrm{rad} / \mathrm{s}$.

Due to the probability of observing highamplitude oscillations in the central area of the plate, vibration of the central point at which the excitation force is applied must be analyzed (this point is named 'mid-point'). With an analogy similar to that in Figure 12, midpoint deflection is presented in Figure 13 for a wide range of excitation frequencies with/without optimum AVSs. According to Figure 13, using optimum AVSs guarantees the suppression of plate vibrations in the midpoint (where it is more susceptible to the external force).

\subsubsection{Advancement of a customized look-up diagram for optimal design of the physical parameters of AVSs over a broad spectrum of excitation frequencies}

In this section, the complex algorithm that was devised in the previous sections is utilized to design AVSs for different harmonic excitations. For this purpose, global optimization procedure of Section 3 is extended to an extensive spectrum of excitation frequencies. A set of 5 identical suppressors is attached to the plate at fixed positions that were found in Section 4.2.3. The excitation frequency varies in the extensive range of $(0<\Omega<250 \mathrm{rad} / \mathrm{s})$ and at each frequency, the optimum stiffness for the set of AVSs is found through two sequential search loops. Figure 14 represents the optimum values of stiffness (for 5 identical suppressors) on a broad spectrum of excitation frequencies. Figure 15 shows the reduction indicator corresponding to the set of suppressors with the stiffness values of Figure 14.

It can be perceived that in the frequency spectrum which contains the first 7 natural frequencies of the circular plate, in this system with $0<\Omega<200$ $\mathrm{rad} / \mathrm{s}$, the optimum value for the stiffness of AVSs is related to the frequency of excitation through a parabolic function. The reason is that the stiffness of AVSs must be set by the relation $k \approx m \Omega^{2}$, which results in the most effective suppression of vibrations. Moreover, by analyzing the presented results in Figure 14 , it can be concluded that for frequencies higher than $\Omega=200 \mathrm{rad} / \mathrm{s}$, the predictions are not valid anymore, because the analysis is confined to the first 7 natural frequencies of the hybrid system. Figure 14 can be used as a look-up diagram where we intend to utilize identical suppressors (with similar mass/stiffness) on plate-shaped foundations in industry.

Mounting the vibration suppressors based on the proposed algorithm and implementing them in an actual framework is however restricted by some technical issues. While it is costly or, in some cases, impossible to provide very high stiffness absorbers in the framework, it is important to consider the frequency of the excitations that are applied to the plate. The results of this section show that stiffness is proportional to the frequency of external load. The design is performed for extreme cases of high-frequency loads while the probability of confronting such conditions is relatively low. 

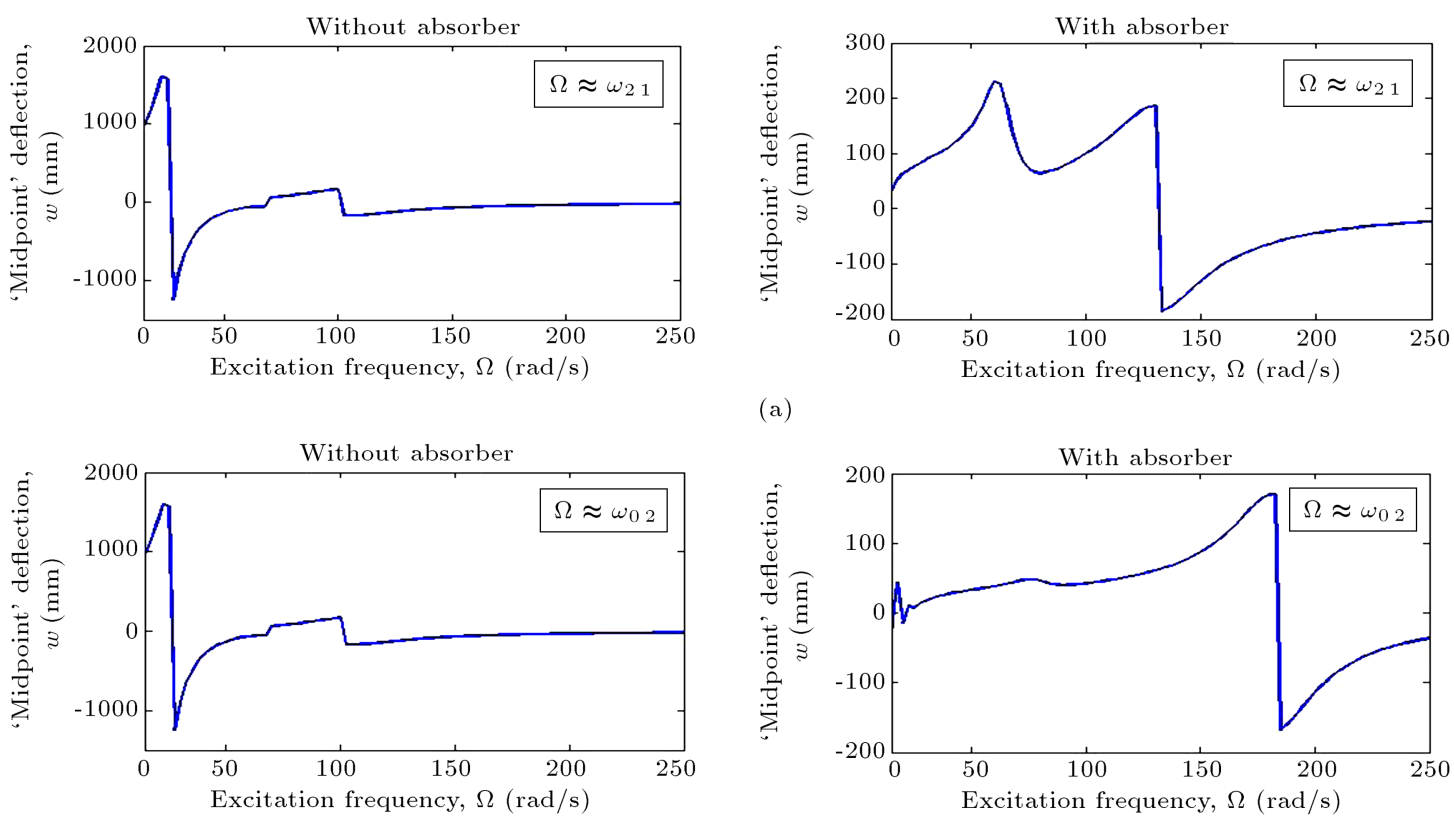

(a)

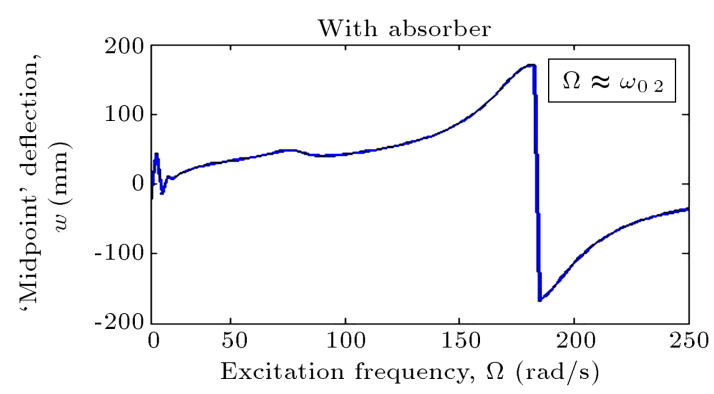

(b)
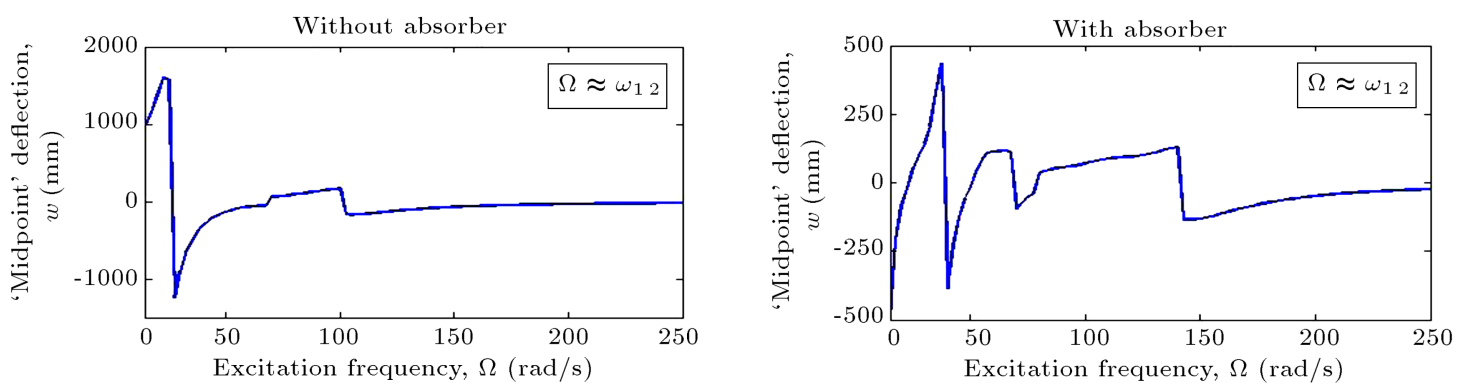

(c)
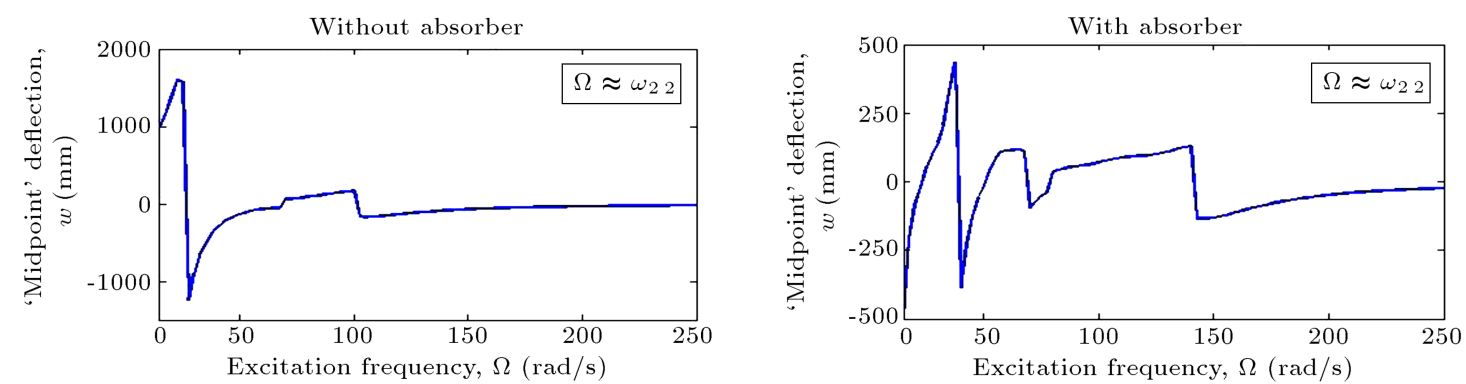

(d)
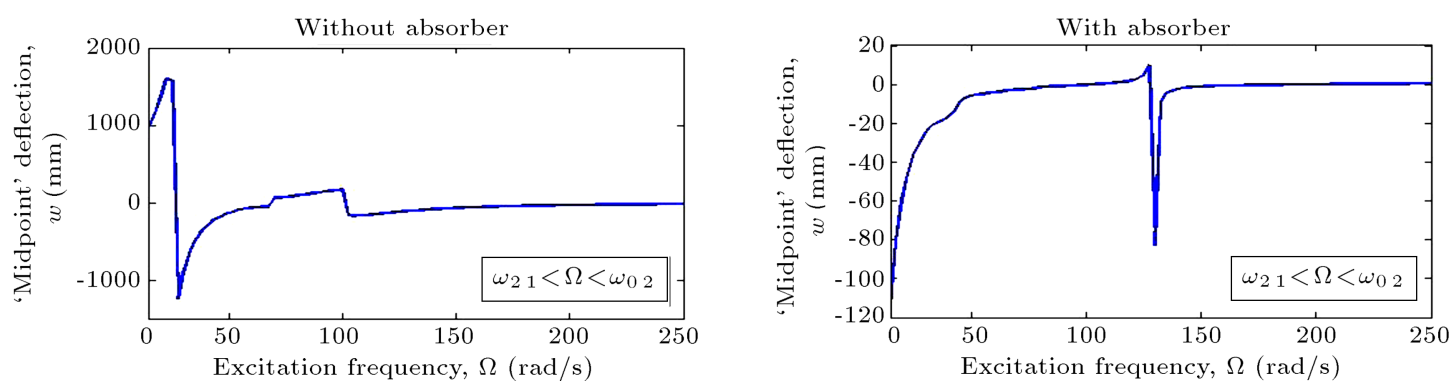

(e)

Figure 13. Midpoint deflection for different values of excitation frequency, without AVSs (left column) and with optimum AVSs (right column); in each case, AVSs designed for $(\mathrm{a}) \Omega \approx \omega_{21}$, (b) $\Omega \approx \omega_{02}$, (c) $\Omega \approx \omega_{12}$, (d) $\Omega \approx \omega_{22}$, and (e) $\omega_{21}<\Omega<\omega_{02}$ are implemented. 


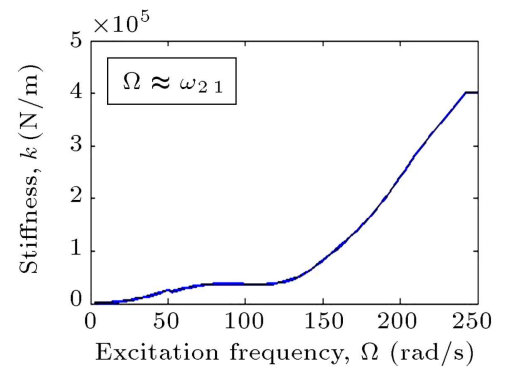

(a)

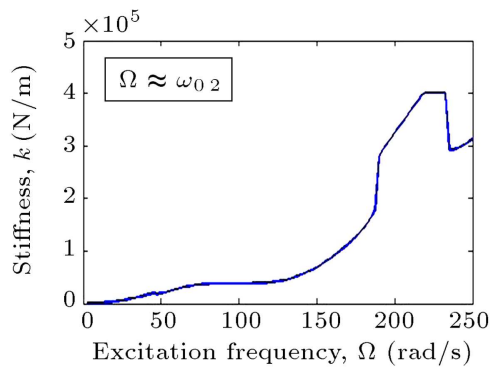

(b)

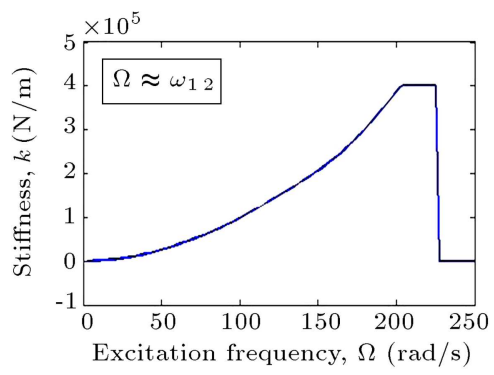

(c)

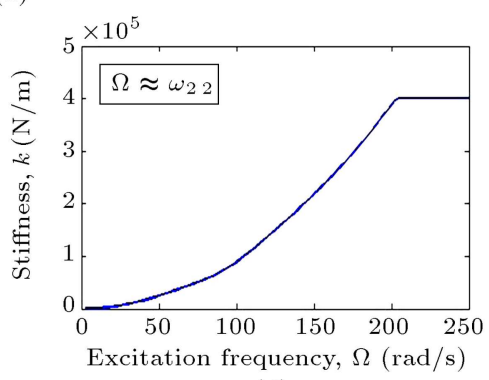

(d)

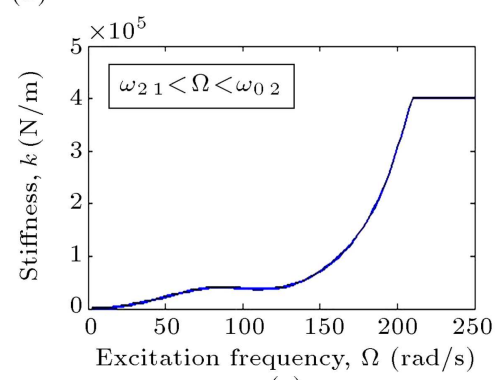

(e)

Figure 14. Optimum stiffness values of AVSs for different excitation frequencies, when the set of identical suppressors are mounted on the optimum locations found in $(\mathrm{a}) \Omega \approx \omega_{21}$, (b) $\Omega \approx \omega_{02},(\mathrm{c}) \Omega \approx \omega_{12},(\mathrm{~d}) \Omega \approx \omega_{22}$, and $(\mathrm{e}) \omega_{21}<\Omega<\omega_{02}$ conditions (locations are given in Table 4).

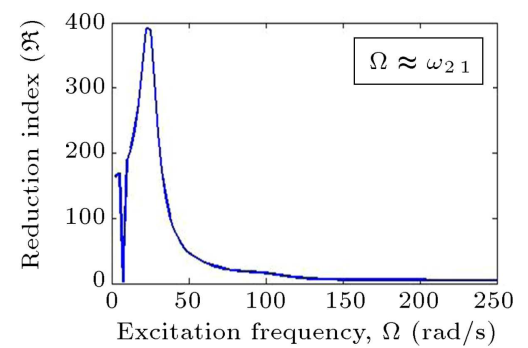

(a)

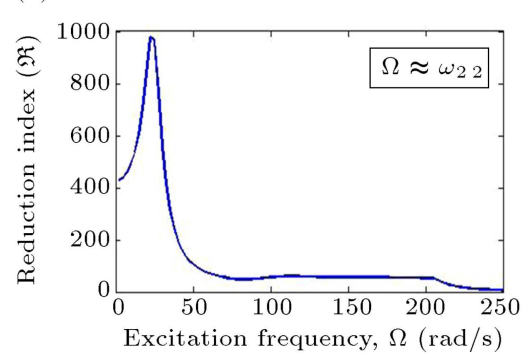

(d)

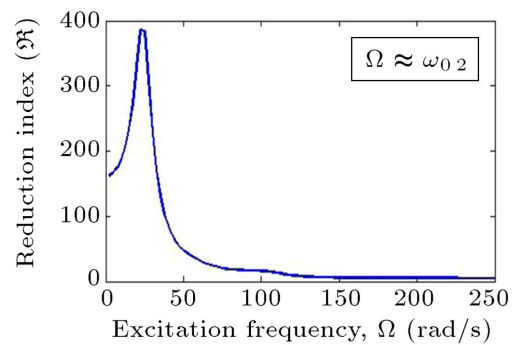

(b)

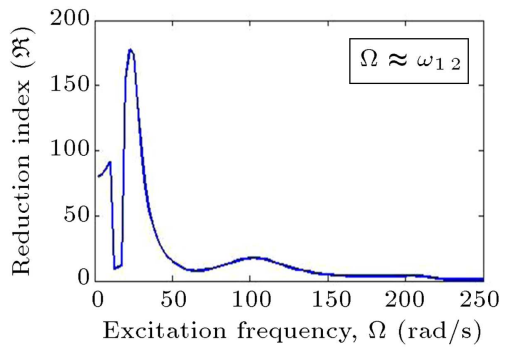

(c)

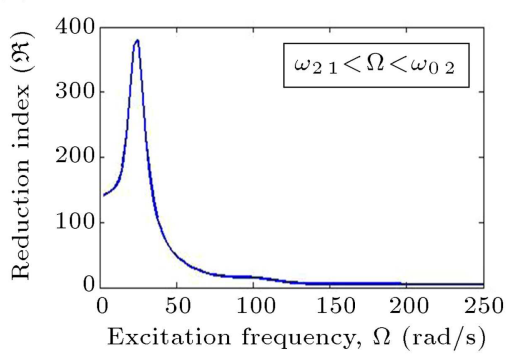

(e)

Figure 15. The values of reduction indicator when the set of identical suppressors are mounted at the optimum locations found in $(\mathrm{a}) \Omega \approx \omega_{21}$, (b) $\Omega \approx \omega_{02},(\mathrm{c}) \Omega \approx \omega_{12},(\mathrm{~d}) \Omega \approx \omega_{22}$, and (e) $\omega_{21}<\Omega<\omega_{02}$ conditions (locations are given in Table 4).

It can be concluded that the designed configurations of AVSs can be utilized for various cases of low and high frequencies, while the cost of employing them depends on the loads that are experienced in each case.

As already mentioned, in Figures 14 and 15, a set of 5 identical suppressors are attached to the plate at fixed positions that were found in Section 4.2.3. These fixed positions are determined for various resonance $\left(\Omega \approx \omega_{21}, \omega_{02}, \omega_{1_{2}}, \omega_{22}\right)$ and non-resonance $\left(\omega_{21}<\right.$
$\Omega<\omega_{02}$ ) cases. Therefore, the last frequency included in this analysis is around $\omega_{22}=96.33 \approx 100 \mathrm{rad} / \mathrm{s}$ Consequently, regarding the valid region of Figure 15, we observe large reduction values for excitation frequencies of $\Omega \leq \omega_{22} \approx 100 \mathrm{rad} / \mathrm{s}$.

\subsubsection{Further discussion of possible validity of the proposed approach in an experimental setup}

In the previous sections, mathematical modeling of the 
system, development of the optimization algorithm, and the effects of implementing the designed AVSs on the circular plate were fully discussed. The simulations that were conducted for examining the effectiveness of the suppressors demonstrated that the undesired vibrations could be eliminated by the application of tuned vibration suppressors. However, the simplifications in solving the dynamic equations and the inherent uncertainties of the dynamic system instigate the idea that an experimental test on the defined system will not necessarily lead to the simulation data. Therefore, an experimental test can reliably determine the effectiveness of the proposed method in suppressing the vibrations.

Moreover, preparing a test body consisting of the circular plate with the given dimensions and a frequency variant force is costly in an academic scale. In this regard, some solutions are provided to lay the groundwork for studying the influence of AVSs in various ranges of excitation frequencies. A reliable test approach should address the problems associated with preparing the circular plate, applying the external force, mounting the vibration suppressors, and measuring the vibration amplitudes. Although manufacturing and establishing a base for a plate as big as 8 meters in diameter is difficult in an academic laboratory, reducing the size of the circular plate to smaller sizes will lead to results that are in correspondence with the fullsize plate. The central harmonic force can be applied using a vibratory shaker placed at the central point of the plate and simple mass and spring structures can be utilized as vibrations suppressors. One important aspect of an experimental test is a reliable method for measuring the vibration amplitudes. Regarding this issue, laser sensors can be utilized to detect the differences in vibration suppression (with a similar mechanism to those implemented in some previous researches such as [38]).

\section{Conclusions}

In this research, Adaptable Vibration Suppressors (AVSs) were designed through an optimized approach to absorb the undesired vibrations in a circular plate that was subject to a central dynamic force. A circular plate with a finite number of vibration suppressors and a centric harmonic force was considered in this regard. The mode summation method was utilized to derive the governing mathematical equations of the system. Mass, stiffness, and position of the AVSs were tuned such that plate deflection was minimized all over the plate. In this regard, a comparison parameter, which showed the Root Mean Square (RMS) value of plate deflection before and after applying suppressors, was defined as reduction indicator $(\Re)$.

To fulfill the optimization, a complex algorithm consisting of several iterative loops was developed in the MATLAB environment in which suppressors with the most efficient configuration (i.e., stiffness and position) were added to the system individually. Three search loops were activated subsequently to find the best stiffness value and the best position for placing the suppressor. The optimization procedure was conducted for a broad spectrum of excitation frequencies including both lower and higher natural frequencies of the plate. The results were presented for 5 resonance/non-resonance cases and analyzed in time and frequency domains.

It should be mentioned that the proposed design algorithm was based on the physical parameters and boundary conditions of the plate as well as the excitation forces applied to it. Hence, the data achieved for the position and stiffness of the absorbers highly depended on the structure and the excitations. In cases that the structure was under the effect of dynamic loads with various frequencies, the suppressors could be displaced to attenuate the vibrations at their maximum extent. Moreover, implementing smart materials in the absorbers provided the means for variable-stiffness absorbing. In other words, implementing passive vibration suppressors that could be displaced or stiffnesstuned enabled a semi-active control of the circular plate that was under external excitation. The vibration attenuating mechanism was adaptive to the changes in the external load characteristics.

According to the results, using optimum AVSs reduces the plate deflection to considerably low values compared to the case without suppressors. In order to make a simpler and more convenient design, low number of optimal suppressors with identical stiffness can also act effectively in attenuating the highamplitude vibrations in resonance/non-resonance conditions. Moreover, according to the frequency response of the hybrid system, it was observed that AVSs would guarantee the vibration suppression over an extensive range of excitation frequencies.

Finally, optimum values for the stiffness of suppressors were presented in a look-up diagram for an extensive range of frequencies. Since the algorithm was developed in a general convenient manner, AVS design could be accomplished for other shapes of the plates with different physical features and various boundary conditions. Using the proposed AVSs design, as a semiactive control approach, led to the great advantages of intuitive clarity, simple design, and reduction in hardware and development cost.

\section{References}

1. Wang, Q., Shi, D., Liang, Q., and Shi, X. "A unified solution for vibration analysis of functionally graded circular, annular and sector plates with general bound- 
ary conditions", Composites Part B: Engineering, 88, pp. 264-294 (2016).

2. Minkarah, I.A. and Hoppmann, W.H. "Flexural vibrations of cylindrically aeolotropic circular plates", The Journal of the Acoustical Society of America, 36(3), pp. 470-475 (1964).

3. Narita, Y. "Natural frequencies of completely free annular and circular plates having polar orthotropy", Journal of Sound and Vibration, 92(1), pp. 33-38 (1984).

4. Kang, W., Lee, N.H., Pang, S., and Chung, W.Y. "Approximate closed form solutions for free vibration of polar orthotropic circular plates", Applied Acoustics, 66(10), pp. 1162-1179 (2005).

5. Leissa, A.W., Vibration of Plates, Vol. SP-160. NASA, Washington, DC: US Government Printing Office (1969).

6. Leissa, A.W. "Literature review: Survey and analysis of the shock and vibration literature: Recent studies in plate vibrations: 1981-85 Part I. Classical Theory", The Shock and Vibration Digest, 19(2), pp. 11-18 (1987).

7. Yamaki, N. "Influence of large amplitudes on flexural vibrations of elastic plates", ZAMM-Journal of Applied Mathematics and Mechanics/Zeitschrift für Angewandte Mathematik und Mechanik, 41(12), pp. 501-510 (1961).

8. Kung, G.C. and Pao, Y.H. "Nonlinear flexural vibrations of a clamped circular plate", Journal of Applied Mechanics, 39(4), pp. 1050-1054 (1972).

9. Tobias, S.A. and Arnold, R.N. "The influence of dynamical imperfection on the vibration of rotating disks", Proceedings of the Institution of Mechanical Engineers, 171(1), pp. 669-690 (1957).

10. Tobias, S.A. "Free undamped non-linear vibrations of imperfect circular disks", Proceedings of the Institution of Mechanical Engineers, 171(1), pp. 691-715 (1957).

11. Williams, C.J.H. and Tobias, S.A. "Forced undamped non-linear vibrations of imperfect circular discs", Journal of Mechanical Engineering Science, 5(4), pp. 325335 (1963).

12. Shi, X., Shi, D., Li, W.L., and Wang, Q. "A unified method for free vibration analysis of circular, annular and sector plates with arbitrary boundary conditions", Journal of Vibration and Control, 22(2), pp. 442-456 (2016).

13. Sridhar, S., Mook, D.T., and Nayfeh, A.H. "Non-linear resonances in the forced responses of plates, Part II: asymmetric responses of circular plates", Journal of Sound and Vibration, 59(2), pp. 159-170 (1978).

14. Ribeiro, P. and Petyt, M. "Non-linear free vibration of isotropic plates with internal resonance", International Journal of Non-Linear Mechanics, 35(2), pp. 263-278 (2000).
15. Ribeiro, P. and Petyt, M. "Geometrical non-linear, steady state, forced, periodic vibration of plates, part II: Stability study and analysis of multi-modal response", Journal of Sound and Vibration, 226(5), pp. 985-1010 (1999).

16. Wu, F., Liu, G.R., Li, G.Y., Cheng, A.G., and He, Z.C. "A new hybrid smoothed FEM for static and free vibration analyses of Reissner-Mindlin plates", Computational Mechanics, 54(3), pp. 865-890 (2014).

17. Haterbouch, M. "Effects of the geometrically nonlinearity on the free and forced response of clamped and simply supported circular plates", PhD Thesis, Universite Mohammed V-Agdal, Rabat (2003).

18. Haterbouch, M. and Benamar, R. "Geometrically nonlinear free vibrations of simply supported isotropic thin circular plates", Journal of Sound and Vibration, 280(3-5), pp. 903-924 (2005).

19. Liou, G.S. "Vibrations induced by harmonic loadings applied at circular rigid plate on half-space medium", Journal of Sound and Vibration, 323(1-2), pp. 257-269 (2009).

20. Kerlin, R.L. "Predicted attenuation of the platelike dynamic vibration absorber when attached to a clamped circular plate at a non-central point of excitation", Applied Acoustics, 23(1), pp. 17-27 (1988).

21. Snowdon, J.C. "Platelike dynamic vibration absorbers", Journal of Engineering for Industry, 97(1), pp. 88-93 (1975).

22. Kirk, C.L. and Leissa, A.W. "Vibration characteristics of a circular plate with a concentric reinforcing ring", Journal of Sound and Vibration, 5(2), pp. 278-284 (1967).

23. Azimi, S. "Axisymmetric vibration of point-supported circular plates", Journal of Sound and Vibration, 135(2), pp. 177-195 (1989).

24. Kunukkasseril, V.X. and Swamidas, A.S.J. "Vibration of continuous circular plates", International Journal of Solids and Structures, 10(6), pp. 603-619 (1974).

25. Avalos, D.R., Larrondo, H.A., and Laura, P.A.A. "Transverse vibrations of a circular plate carrying an elastically mounted mass", Journal of Sound and Vibration, 177(2), pp. 251-258 (1994).

26. Ray, M.C. and Shivakumar, J. "Active constrained layer damping of geometrically nonlinear transient vibrations of composite plates using piezoelectric fiberreinforced composite", Thin-Walled Structures, $\mathbf{4 7}(2)$, pp. 178-189 (2009).

27. Vidoli, S. and Dell'Isola, F. "Vibration control in plates by uniformly distributed PZT actuators interconnected via electric networks", European Journal of Mechanics-A/Solids, 20(3), pp. 435-456 (2001). 
28. Caruso, G., Galeani, S., and Menini, L. "Active vibration control of an elastic plate using multiple piezoelectric sensors and actuators", Simulation Modelling Practice and Theory, 11(5-6), pp. 403-419 (2003).

29. Wu, S.T., Chen, J.Y., Yeh, Y.C., and Chiu, Y.Y. "An active vibration absorber for a flexible plate boundarycontrolled by a linear motor", Journal of Sound and Vibration, 300(1-2), pp. 250-264 (2007).

30. Qiu, Z.C., Zhang, X.M., Wu, H.X., and Zhang, H.H. "Optimal placement and active vibration control for piezoelectric smart flexible cantilever plate", Journal of Sound and Vibration, 301(3-5), pp. 521-543 (2007).

31. Hu, Y.R. and $\mathrm{Ng}, \mathrm{A}$. "Active robust vibration control of flexible structures", Journal of Sound and Vibration, 288(1-2), pp. 43-56 (2005).

32. Wiciak, J. "Modelling of vibration and noise control of a submerged circular plate", Archives of Acoustics, 32(4(S)), pp. 265-270 (2014).

33. Khorshidi, K., Rezaei, E., Ghadimi, A.A. and Pagoli, M. "Active vibration control of circular plates coupled with piezoelectric layers excited by plane sound wave", Applied Mathematical Modelling, 39(3-4), pp. 12171228 (2015).

34. Ji, H., Qiu, J., Badel, A., and Zhu, K. "Semiactive vibration control of a composite beam using an adaptive SSDV approach", Journal of Intelligent Material Systems and Structures, 20(4), pp. 401-412 (2009).

35. Badel, A., Sebald, G., Guyomar, D., Lallart, M., Lefeuvre, E., Richard, C., and Qiu, J. "Piezoelectric vibration control by synchronized switching on adaptive voltage sources: Towards wideband semi-active damping", The Journal of the Acoustical Society of America, 119(5), pp. 2815-2825 (2006).

36. Saadabad, N.A., Moradi, H., and Vossoughi, G.R. "Semi-active control of forced oscillations in power transmission lines via optimum tuneable vibration absorbers: with review on linear dynamic aspects", International Journal of Mechanical Sciences, 87, pp. 163-178 (2014).

37. Meirovitch, L., Principles and Techniques of Vibrations, (1), New Jersey: Prentice Hall (1997).

38. Xie, L., Qiu, Z.C., and Zhang, X.M. "Vibration control of a flexible clamped-clamped plate based on an improved FULMS algorithm and laser displacement measurement", Mechanical Systems and Signal Processing, 75, pp. 209-227 (2016).

\section{Biographies}

Navid Asmari Saadabad received the BSc and MSc degrees in Mechanical Engineering from Sharif University of Technology, Tehran, Iran, in 2014 and 2017, respectively. He started his research on semiactive control of vibrations in mechanical systems and continued his career by applying intelligent optimization and control methods in design and control of dynamic systems. His research interests include intelligent systems, mili-/micro-robotics, and nonlinear and robust control approaches.

Hamed Moradi received the BSc degree in Mechanical Engineering (solid mechanics) from Amirkabir University of Technology in 2005, MSc and PhD in Mechanical Engineering (applied mechanics) from Sharif University of Technology (SUT), Tehran, Iran, in 2008 and 2012, respectively. Currently, he is an Assistant Professor in the Department of Mechanical Engineering, Sharif University of Technology. His current research interests include the modeling of dynamic systems and application of robust, nonlinear, and optimal control methods in various dynamics systems such as manufacturing, bio-engineering, thermofluid industrial processes, and power plant engineering. Also, he is active in the analysis of nonlinear dynamics and chaos in various oscillatory phenomena, especially in two areas of thermo-fluid systems and machining chatter vibrations.

Gholamreza Vossoughi received his $\mathrm{PhD}$ from the Mechanical Engineering Department of University of Minnesota in 1992. Ever since, he has been a faculty member of Mechanical Engineering at Sharif University of Technology. He was with the Manufacturing Engineering and Applied Mechanics Division Directors during 1994-1998; and served as the Graduate Dean of Mechanical Engineering during 1999-2003 and the Dean of Mechanical Engineering Department during 2012-2014. He has also been serving as the founder/director of the Mechatronics Engineering programs at both the Main Campus and the International Campus (Kish Island) of Sharif University of Technology since 2004. 